\title{
Structural Relativity and Informal Rigour
}

\author{
Neil Barton*
}

8 June $2020^{\dagger}$

\begin{abstract}
Informal rigour is the process by which we come to understand particular mathematical structures and then manifest this rigour through axiomatisations. Structural relativity is the idea that the kinds of structures we isolate are dependent upon the logic we employ. We bring together these ideas by considering the level of informal rigour exhibited by our set-theoretic discourse, and argue that different foundational programmes should countenance different underlying logics (intermediate between first- and second-order) for formulating set theory. By bringing considerations of perturbations in modal space to bear on the debate, we will suggest that a promising option for representing current set-theoretic thought is given by
\end{abstract}

*Fachbereich Philosophie, University of Konstanz. E-mail: neil.barton@unikonstanz.de.

${ }^{\dagger}$ I would like to thank Carolin Antos, Andy Arana, John Baldwin, Hans Briegel, Mirna Džamonja, Walter Dean, Monroe Eskew, Ben Fairbairn, José Ferreirós, Sarah Hart, Geoffrey Hellman, Deborah Kant, Juliette Kennedy, Daniel Kuby, Hannes Leitgeb, Julia Millhouse, Moritz Müller, Thomas Müller, Gianluigi Oliveri, Georg Schiemer, Daniela Schuster, Fenner Tanswell, Zeynep Soysal, Jouko Väänänen, Giorgio Venturi, Matteo Viale, Andrés Villaveces, Verena Wagner, John Wigglesworth and audiences in Konstanz, Munich, Paris, and Vienna for helpful discussion. Special mention must be made of Chris Scambler-many ideas in the paper arose out of work on a joint project with him, and I am grateful to him for permission to include them (mistakes made in filling out the details are my own). Two anonymous reviewers provided comments that greatly helped improve the paper, and I am very grateful for their close reading and useful remarks. I am also very grateful for the generous support of the FWF (Austrian Science Fund) through Project P 28420 (The Hyperuniverse Programme) and the VolkswagenStiftung project Forcing: Conceptual Change in the Foundations of Mathematics. 
formulating set theory using quasi-weak second-order logic. These observations indicate that the usual division of structures into particular (e.g. the natural number structure) and general (e.g. the group structure) is perhaps too coarse grained; we should also make a distinction between intentionally and unintentionally general structures.

Keywords - set theory, continuum hypothesis, higher-order logic, informal rigour

\section{Introduction}

Mathematicians are often concerned with elucidating structure. In this paper, I'll examine some issues arising under the following assumption:

(Weak Structuralist Assumption) Part of mathematics and its practice can be understood as isolating and studying different structures.

Why is this assumption 'weak'? Well, the usual statement of structuralism is that mathematics just is the study of structure $1^{1}$ We do not make such a strong claim. Rather, we are just assuming the highly plausible claim that mathematics is at least partly concerned with the specification and study of structure.

Two questions are immediately pertinent:

1. What kinds of different structures are there?

\footnotetext{
${ }^{1}$ A good example here is Shapiro:

For our first (or second) approximation, then, pure mathematics is the study of structures, independently of whether they are exemplified in the physical realm, or in any realm for that matter. (Shapiro (1997), p. 75)
}

Examples can be multiplied (e.g. Resnik (1997), Hellman (1996)). More generally, structuralist ideas have a rich history, appearing in the axiomatic work of Hilbert, Dedekind, and (under one interpretation) Zermelo. A different direction to the mathematical appeal of Structuralism was through the study of abstract algebra and related fields in the work of (among others) Bourbaki, Ore, and Noether (as well as contributions by Hilbert and Dedekind in this field as well), before the emergence of category theory and contemporary structuralist programmes in philosophy. See Corry (2004) for an in depth study of the history, and Reck and Schiemer (2019) for a survey of the state of the art. 


\section{How to we isolate them and/or talk about them?}

The first question is often answered by distinguishing between two kinds of structure; particular and general. Isaacson explains the distinction as follows:

The particularity of a particular structure consists in the fact that all its exemplars are isomorphic to each other. The generality of a general structure consists in the fact that its various exemplars need not be, and in general are not, isomorphic to each other. (Isaacson (2011), p. 21)

Exactly what different branches of mathematics have an underlying 'particular structure' is a contentious issue (we discuss this later). However, almost everyone agrees that we can talk about various kinds of finite particular structure (e.g. the structure of ten objects under some well-order). Normally it is assumed that most of our arithmetical talk is concerned with a particular structure; the standard model of arithmetic 2

General structures, by contrast, are not determined up to isomorphism and include groups, rings, and fields. An example: The group of symmetries on a triangle and the group of integers both possess the general structure of being a group, but the former is finite where the latter is infinite.

It is a somewhat controversial question as to whether these two kinds of structure are of the same ontological kind or not, since particular structures seem more fundamental than general structures in the sense that the latter are properties that the former can possess. We speak, for example, of the particular structure of the integers exemplifying the ring structure or the particular structure of the natural numbers under addition exemplifying the general structure of a monoid 3 Still more concrete are the systems exemplifying particular structures. For example, the face of the clock on my wall (with the usual operations of addition) is a system exemplifying the particular structure of the integers mod 12, which in turn exemplifies the general group structure.

The second question (how we isolate and talk about the different kinds of structure) is then easy in the case of general structures for the Weak Structuralist;

\footnotetext{
${ }^{2}$ See Hamkins (2012) for a dissenting voice that we discuss a bit later.

${ }^{3}$ Isaacson (in Isaacson (2011)) seems to take the view that particular structures are somehow more fundamental, referring to a general structure with no particular instances as "vacuous" (p. 25). Similar remarks can be found in Leitgeb (2020), where unlabelled graphs are taken as the particular 'ground level' structures, and general structures are viewed as higher-order properties or classes of particular structures.
} 
she can simply state the conditions she is interested in for some general structure, and in doing so talks about any particular structures and/or systems that satisfy these conditions. The question is harder for particular structures, since here there is the additional challenge of convincing ourselves that we have isolated a structurally unique entity (at least up to isomorphism ${ }^{4}$ ). If a discipline or syntactic theory has a unique particular structure underlying it, then it is often referred to as a non-algebraic theory or discipline, those with no corresponding particular structure (or a general structure) are called algebraic ${ }^{5}$

One way of tackling the question of when we have isolated a particular structure can be derived from the work of Kreisel (in Kreisel (1967)) and has been taken up subsequently by Isaacson (in Isaacson (2011)). They suggest that we have a process of informal rigour by which we obtain mathematical understanding and isolate different particular structures. The rough idea (which I discuss in more detail below) is that we isolate a particular structure by becoming more rigorous about a topic, and manifest this rigour by providing a categorical axiomatisation.

A categorical axiomatisation is a set of axioms $\mathbf{T}$ which determine a unique model up to isomorphism (i.e. any two models of $\mathbf{T}$ are isomorphic). Where categoricity is concerned, one must talk about different logics. The insight provided by Löwenheim-Skolem Theorem shows that first-order logic cannot provide categorical axiomatisations for infinite structures. It is in the work of Resnik (in particular Resnik (1997)) where we find a notion of structural relativity; the idea that

${ }^{4}$ There is a substantial discussion around whether isomorphism is too strong, and perhaps something weaker like definitional equivalence would be better. We set aside this issue for now, things are complicated enough without opening that can of worms, despite its interest. For an overview, see Button and Walsh (2018), Ch. 5.

${ }^{5}$ We discuss these distinctions in $\$ 1$ below. The algebraic vs. non-algebraic distinction goes back at least to Shapiro (1997) (pp. 40-41). Geoffrey Hellman points out that one might wish to eschew the use of the terms 'particular' and 'general' when discussing structures in favour of only talking about algebraic and non-algebraic theories. For the purposes of this paper, I will talk about both particular/general structures and algebraic/non-algebraic theories, since (a) some authors (e.g. Isaacson (2011)) do use this terminology, (b) locutions like "the integers mod 12 exemplify the group structure" do not seem obviously impermissible, and (c) nothing too much hangs on this distinction for the purposes of the paper: My main aim is to analyse how our thought and language interacts with truth values for different claims-the theorist who wishes to eliminate talk of different kinds of structure is welcome to re-read the paper attending only to claims about truth values rather than the taxonomy of structures. 
the structure isolated for different parts of mathematics depends on the logical resources we consider.

This paper brings together these ideas focussing on set theory as a case study. We argue for the following claims:

1. For different foundational programmes corresponding to different levels of informal rigour, it is reasonable to hold that our set-theoretic thought is underwritten by in a logic stronger than first-order, but weaker than secondorder.

2. This shows that the usual distinction between particular and general structures corresponding to different concepts is more fine-grained than we might have initially thought. There are concepts that correspond to intentionally general structures in that the concept is designed to talk about many nonisomorphic structures. Other concepts correspond to unintentionally general structures, where we do not intend for the structure we talk about to be general, yet we do not pin down a particular structure with our discourse.

3. We have some reason to doubt that we are fully informally rigorous about set theory. Rather, we might hold that our level of informal rigour is partial, and in particular our level of informal rigour is not yet enough to determinate a truth-value for the Continuum Hypothesis $(\mathrm{CH})$.

Here's the plan: $\$ 1$ examines the notion of informal rigour as it appears in Kreisel's 1967 paper and how it relates to the problem of the Continuum Hypothesis. We'll make the distinction between top-down informal rigour concerning particular structures (axiomatisations serve as certifications that informal rigour has been achieved) and bottom-up informal rigour (given an axiomatisation we use it to characterise particular structures). $§ 2$ presents three possible interpretations of informal rigour; a quasi-idealist one, a weakly platonistic one, and a strongly platonistic one (we'll see shortly what I mean by these terms). $\$ 3$ presents the idea of structural relativity. $\S 4$ then examines different states we may be in with respect to informal rigour on the basis of different foundational programmes, and examines some possibilities for axiomatisations of our thought. We develop an assumption of Modal Definiteness; that informal rigour about a certain subject matter should not permit conceptual refinement motivating radically different axiomatisations (given a perturbation in temporal or modal space) and use this to analyse our level of informal rigour. \$5 examines some objections and replies. In responding to possible objections, I develop a quadrilemma for the believer that $\mathrm{CH}$ has a determinate truth value; either (i) we mystically do not go astray when coming to justify new axioms, or (ii) we accept that we cannot justify new 
set-theoretic axioms, or (iii) it is possible to become less precise about the structure we talk about as we come to accept more axioms, or (iv) we have to give up a principle of charity in interpreting set-theoretic claims. Finally $\$ 6$ concludes with some open questions.

\section{Informal rigour and the Continuum Hypothe- sis}

In this section we explain informal rigour and the idea that it might be used to show the existence of particular structures. We'll do this by explaining Kreisel's rough idea, and then formulating a more precise thesis (that particular structures are determined via informal rigour) at the end of the section. We'll also explain how Kreisel thought that his account of informal rigour leads to a determinate truth value for the Continuum Hypothesis 6

Kreisel (in Kreisel (1967)) discusses the notion of informal rigour. This represents a development and refinement of the idea that we work mathematically by examining our intuitive notions and laying down axioms for them. Kreisel expands this thought by arguing that the process is not quite so simple; rather than merely analysing our intuitive concepts, we can become successively clearer about a mathematical subject matter and then manifest this clarity through axiomatisations. He writes:

Informal rigour wants (i) to make this analysis [of intuitive notions] as precise as possible (with the means available), in particular to eliminate doubtful properties of the intuitive notions when drawing conclusions about them; and (ii) to extend this analysis, in particular not

\footnotetext{
${ }^{6}$ Interestingly, it certainly seems like Kreisel held something like the Weak Structuralism. For example, he writes:

if one thinks of the axioms as conditions on mathematical objects, i.e. on the structures which satisfy the axioms considered, these axioms make a selection among the basic objects; they do not tell us what the basic objects are. (Kreisel (1967), p. 165, emphasis original)

Whilst the extent to which Kreisel really was a structuralist (rather than merely provided resources useful to structuralism) is certainly an interesting question, I lack the space to address it fully here.
} 
to leave undecided questions which can be decided by full use of evident properties of these intuitive notions. (Kreisel (1967), pp. 138139)

Kreisel's point is well-taken, and the history of mathematics is replete with notions that were initially unclear but slowly came to be made precise through development and reflection. Examples include ideas of completeness/continuity and denseness (early on these were sometimes confused), the notion of derivative (we will discuss this later in \$5), Cantor's analysis of the size of sets, and indeed the notion of set itself was gradually made clearer. However, whilst Kreisel's remarks are suggestive, he does not provide a detailed account of exactly what informal rigour is like. Largely speaking, he takes it for granted that we know what it is when we see it (at least as far as his Kreisel (1967) is concerned).

Despite this, we can make some progress by examining specific questions:

(1.) What are the targets of informal rigour? ${ }^{7}$

(2.) How do we achieve informal rigour?

(3.) What are the consequences of informal rigour?

For (1.) some taxonomy will be useful. When we talk about mathematical structure, there are several important aspects:

(a) The concepts we employ in thinking about mathematics (I'll refer to these using $C, C_{0}, C_{1}, \ldots$ etc. $]^{8}$

(b) The mathematised natural language(s) we use when speaking about structure(s). We will refer to these as discourses, and denote them by $\left(D, D_{0}, D_{1}, \ldots\right)$.

(c) Different formal mathematical theories $\left(\mathbf{T}, \mathbf{T}_{0}, \mathbf{T}_{1}, \ldots\right)$.

(d) Different mathematical structures, both particular and general $\left(S, S_{0}, S_{1}, \ldots\right)$.

\footnotetext{
${ }^{7}$ I thank Verena Wagner for pressing this question in discussion.

${ }^{8}$ Juliette Kennedy suggests that talk of concepts is too unclear, and we would be better off eliminating this language altogether. I am somewhat sympathetic to this position, and certainly feel that it can sometimes muddy the waters. Despite this, language of this kind is useful for setting up the debate, and so I'll continue to use it here. For the reader who has doubt about the coherence of concept-talk, I suggest that they read all mention of concepts as shorthand for their favourite account of the constituents of thoughts.
} 
(e) Different systems exemplifying structures, which for convenience we'll assume are model-theoretic structures $\left(\mathfrak{M}, \mathfrak{M}_{0}, \mathfrak{M}_{1}, \ldots\right)$.

It is important to be clear about these distinctions if we are to provide a fully worked-out account on Kreisel's behalf. Nowhere is he fully explicit about the matter, but his discussion (and a reasonable understanding of the notion) seems to suggest that informal rigour concerns how the concepts underlying discourses can be refined in coming to be precise about structures. Mathematical practice involves communicating in a mathematised natural language, and how we interpret this language is contingent upon the concepts being employed. For example, the interpretation we ascribe to a computer scientist using the term "set" (in a context where we can have non-well-founded 'sets') is different from the interpretation we would ascribe to a set theorist working in some extension of $\mathbf{Z F C}$. This isn't a contradiction; they are simply employing different concepts with their use of language and mean different things with their usage of the term "set". Correspondingly, there are different ways we could systematise or represent their language formally, and in turn different interpretations of this formal language. At the bottom level, the formal theories representing different pieces of mathematised language can be interpreted (contingent on the concepts employed) as about different kinds of structure.

In the rest of the paper, we will assume that the main target of informal rigour is the concepts we employ when speaking or writing in mathematical language (i.e. discourses). Perhaps there is more to be said here, but I'm happy to make this assumption for the purposes of the paper.

With the targets and rough idea of informal rigour in play, we can begin to address (2.) How do we achieve informal rigour? Kreisel provides four examples? key to each is the idea that we develop informal rigour concerning a concept via working with it in practice. In this way we can develop our intuitions, and come to be rigorous about a notion. This rigour can then be formally codified. Our interest will be especially in his remarks about the difference between the independence of the Parallels Postulate from the second-order axioms of geometry,

${ }^{9}$ These include: (I) analysing the difference between independence results, such as the parallels axiom in geometry and the independence of $\mathrm{CH}$ in set theory (the focus of this paper), (II) the relation between intuitive consequence and syntactic/semantic consequence (here he gives his famous 'squeezing' argument, arguing that the informal notion of consequence can be squeezed between the formal classes of a syntactic derivation in first-order logic and semantic consequence in first-order logic), (III) Brouwer's 'empirical' propositions, and (IV) showing that the use of certain models is a conservative extension of arithmetic. 
and the independence of $\mathrm{CH}$ from the axioms of $\mathbf{Z F C}$.

Concerning the axioms of $\mathbf{Z F C}_{2}{ }^{10}$ Kreisel discusses the following:

Theorem. Zermelo (1930 ${ }^{11}$ Let $\mathfrak{M}$ and $\mathfrak{N}$ be models of $\mathbf{Z F C}_{2}$. Then either:

1. $\mathfrak{M}$ and $\mathfrak{N}$ are isomorphic.

2. $\mathfrak{M}$ is isomorphic to proper initial segment of $\mathfrak{N}$, of the form $V_{\kappa}$ for inaccessible $\kappa$.

3. $\mathfrak{N}$ is isomorphic to proper initial segment of $\mathfrak{M}$, of the form $V_{\kappa}$ for inaccessible $\kappa$.

The core point is the following; whilst there is no full categoricity theorem for second-order set theory $\mathbf{Z F C} \mathbf{C}_{2}$, there is for initial segments. ${ }^{12}$ In particular, many versions of $\mathbf{Z F C}_{2}$ with a specific bound on the number of large cardinals (e.g. "There are no inaccessible cardinals" or "There are exactly five inaccessible cardinals") are categorical.

Concerning this theorem, Kreisel writes:

the actual formulation of axioms played an auxiliary rather than basic role in Zermelo's work: the intuitive analysis of the crude mixture of

${ }^{10} \mathbf{Z F C}_{2}$ denotes the second-order formulation of $\mathbf{Z F C}$, where the Axiom Scheme of Replacement is replaced with a single axiom quantifying over functions, and where the Axiom of Choice is replaced with the second-order claim that the universe can be well-ordered by a class-sized function. A concise presentation, including the quasicategoricity result I discuss, is provided in Hekking (2015).

${ }^{11}$ Shepherdson (1951), Shepherdson (1952), and Shepherdson (1953) take Zermelo (1930)'s proof and clear up a few details. A modern presentations of proofs are available in Hekking (2015) and Button and Walsh (2018), §8A, and a version of Zermelo's proof in modern notation is available in Kanamori (2004). A different method, developed recently by Väänänen and Wang (2015) is to move to a proof-theoretic characterisation of categoricity (so called internal categoricity). We will discuss this move later in \$5 See Button and Walsh (2018) (Ch. 11) for an overview of the internal categoricity results.

${ }^{12}$ One can obtain a full categoricity proof of sorts with further meta-theoretic assumptions. See McGee (1997) for a full categoricity result using urelements. Since the assumptions required for this result are relatively controversial (see e.g. Rumfitt (2015), pp. 273-275) we set it aside here. 
notions, namely the description of the type structure, led to the good axioms: these constitute a record, not the instruments of clarification. (Kreisel (1967), p. 145)

How might we then determine a particular structure according to Kreisel? Abstractly speaking, Kreisel's position might then be described as follows. We begin to work with an informal concept $C$, employing it in some mathematical discourse $D$. Gradually we begin to become clearer about $D$ and $C$ via using them in practice, and developing our intuitions about the subject. Once we are eventually clear about the right concept $C^{\prime}$ underlying $D$ (it is at least possible that $C^{\prime}=C$ here), we will have obtained sufficient precision to lay down a theory $\mathbf{T}$ for $C^{\prime}$, which is categorical in that any system $\mathfrak{M}=\mathbf{T}$ is isomorphic to any other system $\mathfrak{M}^{\prime} \models \mathbf{T}$. In this way, by employing our concept $C^{\prime}$ and using $\mathbf{T}$, we have determined a particular structure $S$ up to isomorphism. In the case of set theory, we can think of the development of the idea of cumulative hierarchy and iterative conception of set after 1900 as yielding some particular set-theoretic structures by 1930 when Zermelo showed his 1908 axiomatisation was categorical. We will refer to the way that we can successively become clear about a concept determining a particular structure, before manifesting this rigour via a categorical axiomatisation as top-down informal rigour.

Top-down informal rigour is a way of coming to be clear about a concept and extracting an axiomatisation that determines a particular structure up to isomorphism. However it is not the only way that we can determine particular structures. Once we have accepted some logical resources and mathematical theory (possibly on top-down grounds) we can use these resources to determine other particular structures. For example, suppose that we have accepted informal rigour concerning the concept natural number and that $\mathbf{P} \mathbf{A}_{2}$ manifests this informal rigour concerning a single unique structure (via the Dedekind categoricity theorem). We can then be informally rigorous about the concept hereditarily finite set, since we can find an interpretation of the hereditarily finite sets in the standard model of $\mathbf{P A}_{2}$, and this interpretation determines the hereditarily finite sets up to isomorphism ${ }^{13}$ But we needn't have been already informally rigorous about the concept hereditarily finite set, we have used other resources to characterise it. We will refer to informal rigour obtained via other accepted resources as bottom-up informal rigour concerning a concept.

${ }^{13}$ The interpretation is via the Ackermann encoding of $\langle H F, \in\rangle$ into arithmetic. Sets are represented by natural numbers, and $n E m$ when the $n^{t h}$ binary digit of $m$ is 1 . $\langle\mathbb{N}, E\rangle$ is then isomorphic to $\langle H F, \in\rangle$. Of course, we can then give a categorical axiomatisation of the hereditarily finite sets using the theory $\mathbf{Z F C}_{2}$-Infinity+" There are no infinite sets". 
This brings us on to (3.) What are the consequences of informal rigour? Our focus will be how informal rigour affects our attitude to the truth value of $\mathrm{CH}$. Key here is the Zermelo quasi-categoricity theorem; this shows that given an interpretation of the second-order variables (this will be important later), $\mathbf{Z F C} \mathbf{C}_{2}$ determines several particular structures corresponding to initial segments of the cumulative hierarchy.

Kreisel took this to show that our talk concerning the cumulative hierarchy, as axiomatised by $\mathbf{Z F C}$, was unambiguous. He writes:

Denying the (alleged) bifurcation or multifurcation of our notion of set of the cumulative hierarchy is nothing else but asserting the properties of our intuitive conception of the cumulative type-structure mentioned above. (Kreisel (1967), pp. 144-145)

Why is this significant for $\mathrm{CH}$ ? Well, since the truth value of $\mathrm{CH}$ is settled by $V_{\omega+2}$ (well below the least inaccessible) and if we think that all models of $\mathbf{Z F C}_{2}$ agree up to the first inaccessible (by the Zermelo quasi-categoricity theorem), then $\mathrm{CH}$ has the same truth-value in all particular structures meeting our informally rigorous concept of set (so the thinking goes). This, as Kreisel points out, makes the independence of $\mathrm{CH}$ from set-theoretic axioms markedly different from the independence of the Parallels Postulate (PP) from the axioms of geometry; PP can have different truth-values across models of the second-order axioms of geometry (once we fix upon some interpretation of the second-order variables), whereas $\mathrm{CH}$ has the same truth value in all models of $\mathbf{Z F C}_{2}$ with the same interpretation of the range of second-order quantifiers.

To make the state of the dialectic precise, and given the difficulty of interpreting Kreisel, it is worth pulling out the key moving parts of our interpretation of Kreisel's presentation:

(Assumption of Informal Rigour) A putatively non-algebraic mathematical discourse $D$ determines a particular structure $S$ when we are informally rigorous in employing the relevant concept $C$ corresponding to $D$, and this informal rigour can be manifested in a categorical axiomatisation $\mathbf{T}$ of $C$ such that for any systems $\mathfrak{M}$ and $\mathfrak{M}^{\prime}$ exemplifying $S$, both $\mathfrak{M}$ and $\mathfrak{M}^{\prime}$ satisfy $\mathbf{T}$ and are isomorphic.

(Manifestation Thesis) We become informally rigorous about a concept $C$ through either (a) developing our mathematical understanding of $C$ by working with it in practice (i.e. top-down rigour), or (b) characterising it through already accepted resources (i.e. bottom-up 
rigour). In the case of concepts for particular structures, this understanding can then be manifested by a categorical axiomatisation T. (In other words, the existence of a categorical axiomatisation is necessary for us to have informal rigour about a concept determining a particular structure.)

(Segment Particularity Thesis) We are informally rigorous about the concept cumulative type structure below the first inaccessible, and this concept is axiomatised by the theory $\mathbf{Z F C}_{2}+$ "There are no inaccessible cardinals" and determines a particular structure.

(CH-Determinateness Thesis) The concept cumulative type structure suffices to determine a truth value for $\mathrm{CH}$.

(Difference Thesis) The kind of independence exhibited by $\mathrm{CH}$ (relative to $\mathbf{Z F C}_{2}$ ) and PP (relative to the axioms of geometry) are of fundamentally different kinds.

In what follows, we shall take the Assumption of Informal Rigour as an assumption (though we'll discuss how to flesh it out in more detail). This is just because I'm interested in exploring the idea; it's clearly a very controversial assumption! We'll argue that the Segment Particularity Thesis and $\mathrm{CH}$-Determinateness Thesis can be challenged. We'll then argue that the Manifestation Thesis suggests that our thought is perhaps best axiomatised by something weaker than $\mathbf{Z F C}$. We'll also argue that the Difference Thesis still holds true.

\section{Three interpretations of informal rigour}

In the last section, we saw some theses that one might extract from Kreisel's paper on informal rigour. In this section, I'll present three ways of interpreting this process of informal rigour that will be important for later.

\subsection{Isaacson's Kreisel}

One way of interpreting the process of informal rigour has been proposed by Dan Isaacson (in Isaacson (2011)). There he seems to commit himself to the Assumption of Informal Rigour in the following passage: 
We achieve understanding of the notion of mathematical structure not by axiomatizing the notion but by reflecting on the development of mathematical practice by which particular mathematical structures come to be understood, the natural numbers, the euclidean [plane], the real numbers, etc. how do we know that such structures exist? The question is likely to be construed in such a way that it is a bad question. There is nothing we can do to establish that particular mathematical structures exist apart from articulating a coherent conception of such a particular structure. (Isaacson (2011), p. 29)

as well as the Manifestation Thesis:

...if the mathematical community at some stage in the development of mathematics has succeeded in becoming (informally) clear about a particular mathematical structure, this clarity can be made mathematically exact. Of course by the general theorems that establish firstorder languages as incapable of characterizing infinite structures the mathematical specification of the structure about which we are clear will be in a higher-order language, usually by means of a full secondorder language. Why must there be such a characterization? Answer: if the clarity is genuine, there must be a way to articulate it precisely. if there is no such way, the seeming clarity must be illusory. (Isaacson (2011), p. 39)

However, his interpretation of these notions is decidedly not objectual in the platonistic sense of concerning mind-independent abstract objects:

The basis of mathematics is conceptual and epistemological, not ontological, and understanding particular mathematical structures is prior to axiomatic characterization. When such a resulting axiomatization is categorical, a particular mathematical structure is established. Particular mathematical structures are not mathematical objects. They are characterizations. (Isaacson (2011), p.38, my emphasis)

So, for Isaacson, the process of informal rigour can be understood as a minddependent activity in some sense. The process of informal rigour should not be understood as one where we pick out some pre-existing ontological objects, but rather as the determination of a particular structure using our thought and language, one that does not exist in advance of our characterising activity (in this sense, his view is quasi-idealist). This precision in our concept is then manifested by a categorical axiomatisation $\mathbf{T}$. 
Isaacson's claim that particular structures just are characterisations is a little puzzling; the claim that particular structures are literally numerically identical with theories (i.e. characterisations) has the whiff of a category mistake about it. However, it serves to show further how we might think of informal rigour as a process of mathematical claims being dependent upon our epistemological and conceptual activity, rather than any independently existing structural domain.

Isaacson's version of informal rigour does not commit him to an 'anything goes' version of conventionalism. First, given some employed concepts about which we are informally rigorous, there can be objective facts about what follows from that concept ${ }^{14}$ This is visible from Isaacson's endorsement of the $\mathrm{CH}$ Determinateness Thesis 15 Moreover, we are able to fix infinitely many structures in this way via bottom-up characterisations ${ }^{16}$ For example, the categoricity of the natural numbers establishes infinitely many particular structures, e.g. the structure exemplified by $(n,<)$ for any chosen $n$. Since it is unclear whether or not Kreisel would have accepted Isaacson's interpretation, I shall refer to a character I call 'Isaacson's Kreisel' as a proponent of this view of informal rigour.

${ }^{14} \mathrm{~A}$ good question, one we do not have space to address here, is how Isaacson's version of Kreisel relates to Ferreirós (2016)'s account of mathematics as invention cum discovery.

${ }^{15}$ He writes:

...the independence of the continuum hypothesis does not establish the existence of a multiplicity of set theories. in a sense made precise and established by the use of second-order logic, there is only one set theory of the continuum. it remains an open question whether in that set theory there is an infinite subset of the power set of the natural numbers that is not equinumerous with the whole power set. (Isaacson (2011), pp. 48-49)

${ }^{16}$ He writes:

While indeed there are up to any given moment of course only finitely many theorems establishing categorical characterizations of structures, e.g. of the natural numbers, the real and complex numbers, the euclidean plane, the cumulative hierarchy of sets up to a particular ordinal, one such theorem may establish categorical characterization of infinitely many particular substructures. (Isaacson (2011), p. 38) 


\subsection{Weak Kreiselian Platonism}

Isaacson's Kreisel represents a version of informal rigour which feeds into a quite anthropocentric characterisation of the notion of structure. On his characterisation, informal rigour concerning the concepts employed in a discourse is constitutive of establishing the relevant structure in question.

Instead, we might have a more platonistic conception of informal rigour. One might rather hold that structures are mind-independent, and there are many abstract concepts we can employ in talking about those structures.

Given a discourse $D$ and employment of a concept $C_{0}$ underlying this discourse, informal rigour on this picture consists of a successive narrowing down and improvement of the concept $C_{0}{ }^{17}$ If $C_{0}$ does not already determine some particular structure $S$, this may then necessitate moving to a sharper concept $C_{1}$ to underwrite $D$. Once we have become sufficiently informally rigorous about the concept underlying $D$ (this might take several iterations of conceptual refinement) and have pinned down some mind-independent particular structure $S$ with some concept $C_{2}$, we are then able to provide our categorical axiomatisation $\mathbf{T}$ corresponding to $C_{2}{ }^{18}$

In many ways, at a practical level, the Weak Kreiselian Platonist and Isaacson's Kreisel have much in common. They both think that mathematics depends in some way on us, the Weak Kreselian Platonist because the ways we refine our concepts are presumably dependent upon us (even though they may be constrained), and Isaacson's Kreisel because mathematical structures are determined by our activity. They differ in that the Kreselian Platonist thinks that the structures we talk about, and plausibly the concepts employ, are independent of us and informal rigour allows us to make a selection between them. Isaacson's Kreisel, on the other hand, thinks that the structures are determined by us, rather than

\footnotetext{
${ }^{17}$ Of course, there may be more than one concept involved, in which case we might have to consider a concepts $C_{0}, \ldots, C_{\alpha}$ instead. I suppress this complication; nothing in my arguments hangs on there being just one concept or many.

${ }^{18}$ One question, that we shall leave as an open question at the end of the paper, is how we should understand this process of conceptual refinement. For example: Do the concepts stay the same, or do they change when we refine our concepts? For the purposes of discussing informal rigour and whether or not $\mathrm{CH}$ is determinate, I'm not sure this matters so much, but for the future development of set theory (and mathematics more generally) we might wonder how conceptual refinement figures in debates about, for example, the temporal continuity of subject matter in mathematics. I am grateful to Chris Scambler for many hours of interesting discussion here.
} 
discovered.

\subsection{Strong Kreiselian Platonism ${ }^{19}$}

There is a stronger version of Kreiselian Platonism. The key additional assumption is the following:

(Set-Theoretic Uniqueness) There is one and only one correct concept $C$ for discourse that is sufficiently 'set-like' (i.e. concerns extensional objects), and it is possible for us to have informal rigour about $C$. Informal rigour should be understood as a way of approximating $C$ ever more closely.

So, for the Strong Kreiselian Platonist, it is not only the case that we may refine concepts in coming to be informally rigorous but also that we tend towards exactly one such way of filling out the concept in the case of set theory.

We then have three figures; Isaacson's Kreisel, the Weak Kreiselian Platonist, and the Strong Kreiselian Platonist. We shall argue that for Isaacson's Kreisel and the Weak Kreiselian Platonist, the status of the informal rigour of the universe of sets (and in particular the Continuum Hypothesis) is questionable. The Strong Kreiselian Platonist can hold on to the full informal rigour of the set concept up to a certain level, but we will argue that their position faces a quadrilemma.

\section{Structural relativity}

We are now at a point where we have said a little more about how we might fill out an account of informal rigour, and provided some possible philosophical interpretations of the notion. For the purposes of our arguments in $\$ 4$ and interpreting our own set-theoretic discourse, it will be useful to set up the idea of structural relativity.

Structural relativity is the idea that the structure isolated by a particular piece of mathematical discourse is contingent upon the logic used to underwrite it. It is discussed explicitly by Resnik (1997):

\footnotetext{
${ }^{19} \mathrm{I}$ am grateful to Leon Horsten for suggesting this interpretation, and Daniel Kuby for some additional discussion led me to realise that I also needed to consider the weaker form of Kreiselian Platonism as discussed in the last subsection.
} 
In thinking about formulating a theory of structures we must take into account a phenomenon I will call structural relativity, the structures we can discern and describe are a function of the background devices we have available for depicting structures ... This relativity arises whether we think of patterns and structures as a kind of mould, format, or stencil for producing instances, or as whatever remains invariant when we apply a certain kind of transformation, or as an equivalence class or type associated with some equivalence relation. The structures we recognize will be relative to our devices for specifying forms, or transformations or equivalence relations. (Resnik (1997), p. 250)

The idea then for Resnik is that the kind of structures we can talk about can vary contingent upon the logical resources we employ. For the same mathematical discourse $D$, we might pick many different formal theories to underwrite it, and many different kinds of structure might be thereby isolated. For example, he writes:

If we limit ourselves to describing structures as the models of various first-order schemata, then the types of structures we will define will be like the more coarse-grained ones frequently found in abstract algebra. Here one starts by defining a type of structure such as a group, a ring, or a lattice with the intention of allowing for many non-isomorphic examples of the same type. As a result most of our structural descriptions will fail to be categorical. On the other hand, using second-order schemata, we can formulate categorical descriptions of the structures studied by (second-order) number theory, Euclidean geometry and analysis, and categorical extensions of $\left[\mathbf{Z F C}_{2}\right]$ that are considered powerful enough for most mathematical needs.

Thus, depending upon our logical resources, we might introduce:

The First-Order Natural Number Structure,

The Second-Order Natural Number Structure,

The First-Order Structure of the Reals,

The Second-Order Structure of the Reals,

and so on.

By going to stronger logics we get more fine grained versions of the various structures. (Resnik (1997), p. 252) 
So, for example, we can consider our talk about natural numbers as either formalised in first-order Peano Arithmetic (PA), or in second-order Peano Arithmetic $\left(\mathbf{P A}_{2}\right)$. The latter axiomatisation corresponds (given the full semantics) to the particular structure of the standard model of natural numbers, the former on the other hand is a general structure that is true both on the standard model (where, presumably, $C$ on (PA) holds), but also can be true in non-isomorphic non-standard models (where, for example, $\neg \operatorname{Con}(\mathbf{P A})$ can hold).

The above passage is fairly indicative of what seems to be a (false) dichotomy underlying parts of the literature; we are presented with the choice between either using first-order resources (where almost nothing is categorical, only finite structures) or full second-order resources (where an enormous amount of our mathematical talk is fully categorical) ${ }^{20}$ This dichotomy does not adequately reflect the fact that in mathematical logic we have a wide range of logics intermediate between first-order and second-order. The properties of these logics are well-understood ${ }^{21}$, and it is surprising that they have not been considered in detail in the context of structural relativity. This is not to say that authors (including Resnik) intend this false dichotomy, just that largely speaking in the structuralist literature these are the two options proffered.

Admitting intermediate logics into interpretations of structural relativity opens up a host of possibilities. Once we free ourselves of the binary choice between first- and second-order resources, we have the option of considering many different formal theories for underwriting a discourse. There is a wide variety of options here, including increasing our resources beyond first-order with certain operators (e.g. ancestral logic) or alternatively allowing infinitary conjunctions or quantifier alternations. Since we will be interested here in theories that we can use in manifesting informal rigour, we set aside the use of infinitary resources. In the next section, we shall see how versions of set theory incorporating structural relativity given by weak second-order logic and quasi-weak second-order logic correspond to two natural positions about informal rigour concerning the cumulative hierarchy.

${ }^{20}$ Isaacson, for example, writes:

As Shapiro and others have long noted, the language in which to articulate our understanding of particular mathematical structures is second-order... (Isaacson (2011), p.28)

${ }^{21}$ See, for example, Shapiro (1991) (Ch. 9) or Shapiro (2001). 


\section{The concept of set, degrees of informal rigour, and structural relativity ${ }^{22}$}

We are now in a position where:

(1.) Informal rigour in the concepts underlying a discourse is manifested by axiomatisations that are categorical, either by top-down or bottom-up approaches.

(2.) We have three different ways of interpreting informal rigour, via Isaacson's Kreisel, Weak Kreiselian Platonism, and Strong Kreiselian Platonism.

(3.) Structural relativity may come in to play, whereby the kinds of structures we isolate are contingent upon the background logic we use.

In this section, I'll consider some examples that show how we might not be fully informally rigorous about our set-theoretic discourse and set concept. I'll then argue that there are reasons to think that there may be a degree of structural relativity involved in the axiomatisation of our thought concerning sets. Nonetheless, I shall argue that we are (and have been) partially informally rigorous, and our discourse about portions of the hierarchy can be understood as about particular structures. To do this, I'll look at a Predicativism proposed by Feferman and Hellman, and then historically at Mirimanoff's thought concerning the Axiom of Foundation, before considering our own axiomatisation of set theory in terms of $\mathbf{Z F C}$ and our possible attitudes to $\mathrm{CH}$.

In order to make out my conclusions, it will be useful first to analyse in a little more detail what we might expect from an account of informal rigour. Important for Kreisel's notion is that our concept of set, and the informal rigour we have about it, is a source for axioms. He writes:

What one means here is that the intuitive notion of the cumulative type structure provides a coherent source of axioms; our understanding is sufficient to avoid an endless string of ambiguities to be resolved by further basic distinctions..23 (Kreisel (1967), p. 144)

${ }^{22}$ This section, and in particular my discussion of what I'll call the Modal Definiteness Assumption, is enormously indebted to Chris Scambler. We worked on this together as part of a joint project, and I am very grateful for his kind permission to include the following discussion in this piece. Of course, any mistakes made in filling out the details should be attributed to me rather than Chris.

${ }^{23}$ Kreisel continues:“...like the distinction above between abstract properties and sets 
Isaacson agrees, at least insofar as interpretation of Kreisel goes:

In order actually to solve the continuum problem a formalizable derivation from axioms, of the kind which Cohen and GödelâĂŹs results show not to exist from the first-order axioms of $\mathbf{Z F}$, must be found. This means that new axioms are required. (Isaacson (2011), p. 16)

My point is the following: If we are informally rigorous about a discourse $D$ and the concepts underlying it, and hence have determined a particular structure, we can expect the use of these concepts as a "coherent source of axioms" not to lead us in radically different directions. Of course, it is possible to have beliefs about a structure that turn out to be false (as when I believe an eventually false conjecture), but it should not be the case that radically different concepts, with radically different theories and consequences are legitimate ways of refining our current concepts. We therefore identify the following:

(The Modal Definiteness Assumption or MDA) If we are informally rigorous about a mathematical discourse $D$, using a concept $C_{0}$ to determine a particular structure $S$, then there should not be two (or more) legitimate ways of refining $C_{0}$ (to some $C_{1}$ and $C_{2}$ ) such that $C_{1}$ motivates a theory $\mathbf{T}_{1}$ and $C_{2}$ motivates a theory $\mathbf{T}_{2}$ such that $\mathbf{T}_{1}$ and $\mathbf{T}_{2}$ are inconsistent with one another ${ }^{24}$

Why do I call this assumption 'modal'? Throughout the rest of the paper, we will consider small perturbations concerning how things might have gone in the past, or might go in the future, and show that given these assumptions an agent's concepts might be expanded in different ways to incompatible extensions. This then casts doubt on the claim that their concept is informally rigorous and determines a particular structure regarding some subject matter.

The Modal Definiteness Assumption is definitely controversial, but also intuitively plausible. If our discourse and concepts already determine a particular structure (via informal rigour) then there should not be equally legitimate ways of sharpening our concepts that are inconsistent with one another, since the truth values of all claims in the discourse are already set by this structure. Therefore one of the two theories has to be false, thus one of the two concept-schemes is

of something.", speaking about the distinction between intensional entities and sets (this intensionality he seems to diagnose as the source of the class-theoretic paradoxes). Since this diagnosis is rather controversial, I'll set it aside here.

${ }^{24}$ Many thanks to Daniela Schuster for pressing me to become clearer about my formulation of the MDA. 
inferior, and so they are not equally legitimate ${ }^{25}$ Of course, what constitutes a 'legitimate' extension is going to be something of debate, but the rough idea is that a change or refinement of a concept is one that still coheres with the original, but adds well-motivated content. Whilst these are difficult ideas to make precise, I hope that examination of the examples I provide from the philosophy of set theory will make it clear that there may be such sharpenings, and hence by the MDA we may not be informally rigorous about our concept of set. However, let us first see how the MDA might play out in a positive case where we do take ourselves to have informal rigour.

\subsection{The Radical Relativist}

Suppose we believe that our discourse about the natural numbers, underwritten by our concept of natural number, is informally rigorous and this informal rigour is manifested by $\mathbf{P A}_{2}$ and the attendant Dedekind-categoricity theorem. Along comes the Radical Relativist who says to us: You cannot be informally rigorous about arithmetic, since there are legitimate consistent extensions $\mathbf{P} \mathbf{A}_{2}+\operatorname{Con}\left(\mathbf{P A}_{2}\right)$ and $\mathbf{P A}_{2}+\neg \operatorname{Con}(\mathbf{P A})_{2}$ of $\mathbf{P} \mathbf{A}_{2}$ that are inconsistent with one another (where $\operatorname{Con}\left(\mathbf{P} \mathbf{A}_{2}\right)$ is the consistency sentence for $\mathbf{P A} \mathbf{A}_{2}$ given the syntactic deduction relation for second-order logi 26 . What should are reaction be?

Our response should be the following: Of course these extensions are formally consistent, in the sense that assuming $\mathbf{P A} \mathbf{A}_{2}$ is $\omega$-consistent (given the incomplete syntactic deduction relation for second-order logic) a contradiction is not derivable in either $\mathbf{P A}_{2}+\operatorname{Con}(\mathbf{P A})_{2}$ or $\mathbf{P A}_{2}+\neg \operatorname{Con}(\mathbf{P A})_{2}$. One is nonetheless clearly legitimate where the other is not. In particular, $\mathbf{P A} \mathbf{A}_{2}+\neg C$ on $(\mathbf{P A})_{2}$ can only be true in models that are non-standard, both in that (i) the interpretation of the secondorder variables has to be given by a Henkin semantics that permits a two-sorted first-order characterisation, and (ii) the theory also has consequences (assuming $\mathbf{P A}_{2}$ is in fact consistent) that do not accord with our concept of natural number, for example models of the theory contain a natural number $n^{*}$, such that for any particular standard natural number $n$ given to me, $n^{*}$ is greater than $n$. So it is simply not true that $\mathbf{P} \mathbf{A}_{2}+\operatorname{Con}\left(\mathbf{P} \mathbf{A}_{2}\right)$ and $\mathbf{P} \mathbf{A}_{2}+\neg \operatorname{Con}\left(\mathbf{P} \mathbf{A}_{2}\right)$ are both legitimate extensions of $\mathbf{P} \mathbf{A}_{2}$, at least insofar as axiomatising our concepts and thought concerning the particular structure of natural numbers is concerned.

\footnotetext{
${ }^{25}$ If you're familiar with debates in the philosophy of set theory, you might already see where I'm going here.

${ }^{26}$ This will, of course, not be complete. Nonetheless one can define this relation, see Button and Walsh (2018).
} 
Moreover, there is no categoricity theorem for the theory $\mathbf{P A} \mathbf{A}_{2}+\neg \operatorname{Con}\left(\mathbf{P} \mathbf{A}_{2}\right)$, and indeed it can have highly non-isomorphic models. In fact, since we must allow non-full Henkin interpretations here, we are effectively working in a twosorted first-order framework, and so the usual trappings of first-order logic apply. So there can be no categoricity theorem for this theory, and hence no informal rigour.

This will provide a contrast case for our main examples; considering a Predicativism proposed by Feferman and Hellman, examining the historical situation with respect to Miramanoff and the Axiom of Foundation, and our contemporary situation with respect to set theory and $\mathrm{CH}$.

\subsection{The Predicative Iterabilist}

We now consider a slightly different situation, one in which we have agents whose thought is best axiomatised by a version of set theory intermediate between first and second-order ZFC.

Suppose that one accepts that we are informally rigorous about the concept of natural number, but has extreme reservations about the whole of set theory. A view providing a predicative foundation for arithmetic has been advanced by Feferman and Hellman in a pair of papers Feferman and Hellman (1995) and Hellman and Feferman (2000) ${ }^{27}$ In Feferman and Hellman (1995) they define a system EFSC (for Elementary theory of Finite Sets and Classes) and provide a categoricity proof for natural number systems within EFSC. Suppose further that a Predicativist of the Feferman-Hellman variety expands their concepts and accepts the iterative conception as a conceptual idea, and hence regards $\mathbf{Z F C}$ as a (probably) consistent theory worthy of study, but has extreme reservations about informal rigour concerning the notions of arbitrary subset and arbitrary wellorder. Instead, they think that we can only be informally rigorous about things that are predicatively defined, and think that it's possible that our thinking might not be informally rigorous and fail to determine particular structures at large infinite ordinals. Call this character the Predicative Iterabilist. What should the Predicative Iterabilist say about our set-theoretic thought concerning the iterative conception?

To make our points (here and later) we first need to set up some terminology. Two background logics will be of special interest for us ${ }^{28}$

\footnotetext{
${ }^{27}$ I am grateful to Geoffrey Hellman for pointing to the position of Feferman and Hellman as a possible case study.

${ }^{28}$ The presentations given here are heavily indebted to Shapiro (2001).
} 
Definition. Weak second-order logic is the logic in which we allow the same vocabulary as second-order logic $\mathscr{L}_{K}^{2}$ (where $K$ are the non-logical symbols) but with function variables removed.

Its semantics is given by letting the second-order quantifiers range over finite relations. Let $\mathfrak{M}$ be a model with domain $M$. We define a finite assignment $s$ on $\mathfrak{M}$ as assignment $s$ that assigns a member of $M$ to each first-order variable, and a finite $n$-place relation on $M$ to each $n$-place relation variable. Satisfaction is defined in the usual manner for the first-order connectives and quantifiers, and second-order quantification is handled by the clause:

$\mathfrak{M}, s \models \forall X \phi$ iff for every finite assignment $s^{\prime}$ that agrees with $s$ (except possibly at $X), \mathfrak{M}, s^{\prime} \models \phi$.

The instances of Comprehension $\exists X \forall y(X(y) \leftrightarrow \phi(y))$ which are valid on a structure $\mathfrak{M}$ are those where the extension of $\phi$ is finite in $\mathfrak{M}$.

Let $\mathbf{Z F C}_{2 W}$ be set theory formulated in weak second-order logic with instances of the replacement scheme for each formula of the weak second-order language.

Definition. Quasi-Weak Second-Order Logic is the same as Weak Second-Order Logic, but in the semantics each variable assignment assigns countable relations to the variables (i.e. we assign countable relations instead of finite ones). So $\forall X \phi$ holds iff for all countable $X, \phi$ holds.

Let $\mathbf{Z F C}_{2 Q W}$ be set theory formulated in quasi-weak second-order logic with instances of the replacement scheme for each formula of the quasi-weak secondorder language.

It is useful to identify some facts off the bat ${ }^{29}$

Fact. Both $\mathbf{Z F C}_{2 Q W}$ and $\mathbf{Z F C}_{2 W}$ are able to characterise categorically the natural numbers (i.e. any two models of $\mathbf{Z F C}_{2 Q W}$ and $\mathbf{Z F C}_{2 W}$ always have the standard natural numbers as their standard model of arithmetic, and indeed any two

\footnotetext{
${ }^{29}$ See Shapiro (2001) for discussion of these results.
} 
models of $\mathbf{P A}_{2}$ with the full semantics within a model of $\mathbf{Z F C}_{2 Q W}$ or $\mathbf{Z F C}_{2 W}$ are isomorphic). This is because we can characterise the notion of finiteness in both quasi-weak and weak second-order logic ${ }^{30}$ The same goes for the rational numbers 31

Fact. $\mathbf{Z F C}_{2 Q W}$ is able to characterise the theory of real analysis up to isomorphism. Essentially, this is because we can characterise the completeness principle for the reals in $\mathbf{Z F C} 2 Q W{ }^{32}$ In $\mathbf{Z F C} \mathbf{C}_{2 W}$, however, one cannot characterise the reals up to isomorphism, since the Löwenheim number of Weak Second-Order Logic is $\aleph_{0} 33$

Fact. $\mathbf{Z F C}_{2 Q W}$ is able to characterise the notion of well-foundedness, that is, all models of $\mathbf{Z F C}_{2 Q W}$ are well-founded ${ }^{34}$

Fact. $\mathbf{Z F C}_{2 W}$ is not able to characterise the notion of well-foundedness (i.e. there are models of $\mathbf{Z F C}_{2 W}$ with a non-well-founded membership relation) ${ }^{35}$

These facts show that quasi-weak second-order logic has substantially more expressive power than weak second-order logic; we can characterise more notions within in it (and in turn, the versions of set theory formulated in the respective logics differ in their expressive power and intended models).

So, we have several logics and versions of ZFC-like set theory rendered in them in view. Now, the Predicative Iterabilist will hold that we are informally rigorous about the natural numbers, but have grave worries about our informal

${ }^{30}$ See Shapiro (2001), p. 161, and Theorem 16 and Corollary 17 on p. 162.

${ }^{31}$ This is because we can characterise the notion of minimal closure in the two logics, and the rational numbers can be characterised up to isomorphism as an infinite field arising from the minimal closure of $\{1\}$ under the field operations and their inverses. See Shapiro (2001), p. 161.

${ }^{32}$ See Shapiro 1991), pp. 164-165.

${ }^{33}$ See Shapiro (2001), pp. 161-162.

${ }^{34}$ Assuming Choice in the meta-theory, the fact that every countable class is a set in a model of $\mathbf{Z F C}_{2 Q W}$ ensures this. See Shapiro (1991), p. 165.

${ }^{35}$ This is because there is a natural equivalence between being a model of $\mathbf{Z F C}_{2 W}$ and being an $\omega$-model of ZFC (see Shapiro (1991), p. 162, Corollary 17) and there are non-well-founded $\omega$-models of ZFC. 
rigour concerning the iterative conception in general. In this case, we might think that our thought about ZFC-based set theory and the concept of cumulative type structure is best axiomatised by $\mathbf{Z F C}_{2 W}$. There we are able to identify the rational and natural numbers up to isomorphism, but the real numbers cannot be so identified, and various large well-orderings (e.g. $\omega_{1}^{C K}$ ) cannot be characterised up to isomorphism. 36

If you are a Predicative Iterabilist, you are thus likely to hold that our talk about the concept cumulative type structure is only partially informally rigorous, and this level of partial informal rigour is manifested in $\mathbf{Z F C} \mathbf{C}_{2 W}$. We thus have a coherent position on which a level of informal rigour is manifested in a logic stronger than first-order but weaker than second-order.

We can make out this point using the MDA ${ }^{37}$ If I am a Predicative Iterabilist I believe I have grounds for the determinacy of thought concerning the natural numbers, but not the full real numbers, impredicatively defined. How can they make the grounds for this indeterminacy precise using the MDA? Well, they accept the use of $\mathbf{Z F C}_{2 W}$ as underwriting our theory of sets by recognising as absolute the finite sets of a given set, and the natural numbers as determinate. This framework supports informal rigour regarding the concept finite subset of the natural numbers, and this is enough to pin down the natural numbers up to isomorphism. But when we look to expand our theory to the real numbers this framework can be extended in two different incompatible ways. On the one hand, we can extend our determinate theory of the natural numbers to the classical continuum via Dedekind-cuts or equivalence classes of Cauchy sequences in the rationals. On the other hand, we could extend to the intuitionistic continuum, as developed by Brouwer, Heyting and others. These two extensions formally contradict one another; for example the intutionistic theory proves that all functions are (uniformly) continuous, whereas in the classical continuum we have many discontinuous functions ${ }^{38}$ Thus, the Predicative Iterabilist can spell out why she does not think that there is informal rigour concerning the reals in terms of the MDA. Moreover, if we restrict the discussion to the classical continuum, there are

\footnotetext{
${ }^{36}$ See here Shapiro (1991), p. 163.

${ }^{37}$ I am grateful to Geoffrey Hellman for suggesting this as a possible objection to my final position that $Z F C_{2 Q W}$ is a plausible candidate to underwrite our discourse involving sets. By re-purposing the objection to the case of the Predicative Iterabilist, I think that it bolsters the role of the MDA in making precise grounds for indeterminacy.

${ }^{38}$ Examples can be multiplied. A simpler example is the intuitionistic theorem that it is not the case that any given infinite sequence of $0 \mathrm{~s}$ and $1 \mathrm{~s}$, the sequence is either composed of 0 s everywhere or contains a 1 somewhere, contradicting the obvious classical fact. See Dummett (1977), Ch. 3, for a proof.
} 
still different ways of extending her theory $\mathbf{Z F C}_{2 W}$; we might choose to include or exclude axioms of definable determinacy. She also has a quick explanation of why the MDA does not speak against her belief in determinateness concerning the natural numbers; there are no known legitimate expansions of her concept of natural numbers that motivate inconsistent theories. As mentioned in $\$ 4.1$. our concept of natural number clearly excludes known independent sentences (like Gödelian diagonal sentences) as being theory expansions concerning a legitimate conceptual refinement.

Later (\$4.4) we shall see that a similar argument can be made for the believer that the reals are determinate, assuming that we accept axioms of definable determinacy (axioms with close relationships to large cardinals). Of course, the believer in the Segment Particularity Thesis on the basis of the quasi-categoricity of $\mathbf{Z F C}_{2}$ will reject this application of the MDA. We will discuss the place of the quasi-categoricity theorem later (\$5), for now we just note that the example as presented shows that we can have a coherent position on which our reasoning is axiomatised by a set theory couched in a logic intermediate between first- and second-order and this belief can be made precise on grounds involving the MDA. Before we discuss $\mathrm{CH}$, we will mention a historical example.

\subsection{Miramanoff's Informal Rigour}

The following example will provide an example where we have a failure of informal rigour on the basis of the MDA, but might nonetheless think that substantial parts of mathematics are informally rigorous, and as such we have partial informal rigour in the notion of set. We'll see, however, that the example is more analogous to PP than $\mathrm{CH}$ (the latter we consider in \$4.4).

In 1917, Dimitry Mirimanoff wrote a paper entitled 'Les antinomies de Russell et de Burali-Forti et le problème fondamental de la théorie des ensembles'. In this paper, he considers Russell's Paradox and the Burali-Forti Paradox, and identifies two kinds of sets; the 'ordinary' ones and the 'extraordinary' ones. These were to be differentiated by whether or not they contain infinite descending sequences of membership; the ordinary ones do not (in current terminology: they have a well-founded membership relation) and the extraordinary ones do (in current terminology: they have a non-well-founded membership relation):

I will say that a set is ordinary just in case it gives rise to finite descents, I will say that it is extraordinary when among its descents are some that are infinite. (Mirimanoff (1917), p. 42, my translation) ${ }^{39}$

\footnotetext{
${ }^{39}$ The original French reads:
} 
It is clear that Mirimanoff (in Mirimanoff (1917)) was undecided about whether the Axiom of Foundation was a basic principle about sets. It is also fairly clear, we think, that he was not fully informally rigorous about set theory. To see this, it suffices to consider what theory might have underwritten his thinking about sets, and show that there are different legitimate extensions that are inconsistent with one another.

Clearly Mirimanoff thought that sets were extensional and he explicitly discusses the axioms of pairing and union, as well as replacement. For the purposes of our discussion, let us assume that he was clear that his notion of set supported at least the first-order axioms of $\mathbf{Z F}$ without the Axiom of Foundation. (It doesn't matter so much whether or not these were actually Mirimanoff's views, as long as this character is at least possible it shows the kinds of situations that are compatible with informal rigour in set theory.)

Can Mirimanoff's level of informal rigour support more? Is he informally rigorous about the Axiom of Foundation? We answer this negatively using the Modal Definiteness Assumption. We argue that there are legitimate extensions of Mirimanoff's concept that support inconsistent theories of sets (such as ZF and ZF-Foundation+AFA) ${ }^{40}$. Clearly the former is a legitimate extension, since it is what we (as a matter of fact) use now on the basis of our concept of cumulative type structure. Is the latter a legitimate extension of ZF-Foundation? One might be tempted to answer no: The iterative conception of set clearly prohibits the existence of non-well-founded sets.

The iterative conception is emphatically not Mirimanoff's conception of set, however. Whilst he has the concept of ordinal and rank in play 41 it is not really until Zermelo (in Zermelo (1930)) that we start to see the idea of cumulative type structure emerge, solidified in Gödel's work on $L$ (in Gödel (1940)), and it was not until the late 1960s and 1970s that the idea of the iterative conception and its relation to ZFC were fully isolated ${ }^{42}$ Indeed, Mirimanoff seems to treat non-wellfounded sets as legitimate objects worthy of study, formulating a specific notion

Je dirai qu'un ensemble est ordinaire lorsqu'il ne donne lieu qu'a des descentes finies; je dirai qu'il est extraordinaire lorsque parmi ses descentes il y en a qui sont infinies. (Mirimanoff (1917), p. 42)

${ }^{40}$ Here AFA denotes Aczel's Anti-Foundation Axiom, which has strong affinities with the graph conception of set. See Aczel (1988).

${ }^{41}$ The notion of ordinal recurs throughout his discussion of the Burali-Forti Paradox, and he discusses the notion of rank on p. 51 of Mirimanoff (1917).

${ }^{42}$ In Boolos (1971), for example. See Kanamori (1996) for a thorough discussion of the history. 
of isomorphism known as tree-isomorphism that works for both non-well-founded and well-founded sets ${ }^{43}$ The following situation is then possible: Suppose that instead of the iterative conception becoming the default conception of set, the graph conception of set (on which sets are viewed as given by directed graphs) became the default set-theoretic conception. We might, for example, have been persuaded by considerations about non-well-founded sets emerging in computer science (as when they are used to model concurrent processes). ${ }^{44}$ Then, it seems reasonable to accept that Mirimanoff's intellectual descendants would have accepted that there were non-well-founded sets. By the MDA, he can't then have been fully informally rigorous, since there are inconsistent ways of extending the concept he was employing about his discourse.

It is then tempting to say that Mirimanoff's thinking might be best captured by first-order ZF without Foundation. We should resist this temptation. Mirimanoff's context is plausibly one in which he was informally rigorous about what the natural numbers were, and indeed his work comes after Dedekind's categoricity proof (in Dedekind (1888)). In particular, his definition of well-foundedness depends on the notion of finiteness; he characterises well-founded sets as those which only have finite descending membership chains, rather than using the contemporary first-order statement of the Axiom of Foundation in terms of the claim that every non-empty set $A$ contains a set $B$ such that $A \cap B$ is empty (a formulation which appears in Zermelo (1930) ${ }^{45}$. But, by the Compactness Theorem, finiteness cannot be characterised using first-order logic, nor can the natural numbers ${ }^{46}$ It is overwhelmingly likely that he would have not accepted non-standard models of arithmetic as legitimate interpretations in the same sense as his own.

Since Mirimanoff was also well aware that arithmetic could be coded in set theory, we are at a point where we would like to say that his discourse about parts of set theory such as the natural numbers and finite sets are informally rigorous and determined a particular structure. It is also plausible (putting aside worries of Predicativism) that he was informally rigorous around 1915 about the notion

${ }^{43}$ See Aczel (1988), p. 105.

${ }^{44}$ See here Incurvati (2014) for a description of the graph conception and Aczel (1988) for a summary of non-well-founded sets (as well as some useful historical remarks in Appendix A).

${ }^{45}$ See here Aczel (1988), p. 107. Independently, von Neumann presented this formulation in 1929.

${ }^{46}$ In fact, being able to capture these two notions is roughly equivalent, since " $x$ is finite" can be parsed in terms of being bijective with a standard natural number, and " $x$ is standard natural number" can be parsed as being a finite successor-distance away from 0. See Shapiro (2001) (p. 155) for the details. 
of real number, by this stage he was working on the intellectual foundations that had already been laid by Cauchy, Weierstrass, Cantor, and Dedekind, and the categoricity of the real line had been proved. However, by the MDA, his discourse about set theory in general was not informally rigorous. Thus, if we are to provide an axiomatisation for underwriting his discourse and concept of set, we should use a theory and logic that is not fully categorical, but nonetheless can identify parts of set theory up to isomorphism.

What should we say about Mirimanoff's level of informal rigour? Well, to review:

(1.) His concept of set did not clearly support the Axiom of Foundation.

(2.) It is highly plausible that he was informally rigorous about the natural numbers and the real numbers.

(3.) It is highly plausible that he was informally rigorous about the concept of well-order (being able to distinguish and talk about the extraordinary and ordinary sets).

We can then say that Mirimanoff's level of informal rigour about set theory can be roughly characterised by $\mathbf{Z F C}_{2 Q W}$-Foundation (i.e. $\mathbf{Z F C}_{2 Q W}$ with the Axiom of Foundation removed). There, we can characterise the usual objects of mathematics including the real, rational, and natural numbers (since the categorical characterisations of these theories do not depend on the Axiom of Foundation). Moreover, he can formulate and discuss his worries about wellfoundedness in this logic. However, he is not fully informally rigorous, since there are incompatible legitimate expansions of the concept he was working with (namely to one supporting the foundation axiom and to one supporting its negation).

We should remark though that Mirimanoff's situation is more like the situation we have with the Axiom of Parallels in geometry, rather than what we have in $\mathbf{Z F C}_{2}$ with respect to $\mathrm{CH}$. This is because there is no categoricity proof for $\mathbf{Z F C}_{2}{ }^{-}$ Foundation as there are models of $\mathbf{Z F C}_{2}$-Foundation in which Foundation holds and others in which it fails. So whilst our example shows that there might have been a case where we failed to be informally rigorous about our notion of set, it does not yet show the possibility of a situation where we are not, where we have the iterative conception of set.

\subsection{Modal Definiteness and the Continuum Hypothesis}

So then: What now about our own thought concerning the Continuum Hypothesis? My contention is that, given the Modal Definiteness Assumption, we have 
good reason to think that we are not fully informally rigorous about our concept of set. To see this, it is useful to consider two active programs targeting the resolution of $\mathrm{CH}$ in the contemporary foundations of set theory, namely forcing axioms and Woodin's Ultimate- $L$ programme.

We omit the details here, since they are technically rather tricky, and many questions are still open. A rough description of each, however, will help to see the senses in which they present legitimate conceptual refinements of our concept of cumulative type structure. Both kinds of programme attempt to capture notions of 'maximality' in some way. Ultimate- $L$ does so by incorporating large cardinals in an elegant manner, potentially providing a model in which many questions are decidable but large cardinals can also exist ${ }^{47}$ Forcing axioms on the other hand ensure that various kinds of subset exist; in technical terms, they assert that the universe has already been saturated under the existence of generic filters for certain partial orders and families of dense sets. Both represent somewhat different takes on how our concept of set may develop; Ultimate- $L$ focusses on the development of large cardinals and inner model theory, whereas forcing axioms try to capture the idea of a rich process of subset formation.

Crucially, if we take the Ultimate- $L$ approach, we can prove $\mathrm{CH}$, and strong forcing axioms such as the Proper Forcing Axiom (PFA) imply $\neg \mathrm{CH}$. They therefore represent inconsistent extensions of our current best theory of sets. They also both seem legitimate; both correspond to natural ways we might develop our set concept.

Given the MDA, it seems then that we are not fully informally rigorous about our concept of set. It is also plausible, however, that we have a good deal of informal rigour. We seem to have informal rigour about the natural numbers, where the only known independent statements are all equivalent to consistency statements, and the negation of these are illegitimate extensions (assuming that we think the axioms really are consistent). For second-order arithmetic, under both Ultimate- $L$ and PFA there are no obvious analogues of $\mathrm{CH}$; both programmes imply Projective Determinacy and there are no known sentences of ZFC independent from the theory $\mathbf{Z F C}$-Powerset $+V=H\left(\omega_{1}\right)$ (other than Gödelian-style diagonal sentences). It also seems clear that our concept of cumulative hierarchy supports the idea that we are informally rigorous about the claim that all sets are

\footnotetext{
${ }^{47}$ Whether we can construct Ultimate- $L$ depends crucially on several conjectures in inner model theory. See Woodin (2017) for details. The key point is that if we are able to build a model that is ' $L$-like' and contains a supercompact cardinal, such a model would be able to tolerate all known large cardinal axioms that are also consistent, in contrast to the situation with $V=L$ and measurable cardinals (assuming that the existence of a measurable cardinal is, in fact, consistent).
} 
well-founded.

Given this, it seems that our level of informal rigour in the cumulative hierarchy of sets might be top-down manifested by $\mathbf{Z F C}_{2 O W}$. In quasi-weak secondorder logic (and hence $\mathbf{Z F C} \mathbf{C}_{2 Q W}$ ) one can (bottom-up) ${ }^{48}$

(1.) Characterise $H\left(\omega_{1}\right)$ up to isomorphism by the theory consisting of:

(i) Extensionality

(ii) The axiom "Every set is countable".

(iii) The Axiom of Foundation, expressed as the claim that there is no $\omega$ length infinite descending $\in$-sequence.

(iv) The sentence in quasi-weak second-order logic expressing "every countable subclass of the domain of discourse is the extension of a set".

This further bolsters our earlier claim that $\mathbf{Z F C}_{2 Q W}$ underlies our settheoretic thought, since (given Projective Determinacy) no MDA-style argument is forthcoming for $H\left(\omega_{1}\right)$.

(2.) The field of reals $(\mathbb{R},+, \times,<)$ is the only model (up to isomorphism) of the theory of ordered fields with the sentence of quasi-weak second-order logic expressing the claim that all Cauchy sequences converge and the Archimedean property that for every $x$ in the domain of discourse, there is a finite sequence $\left\langle y_{i} \mid 0 \leq i \leq n\right\rangle$ of elements of the domain such that $x<y_{n}$, $y_{0}=1$, and for all $i<n, y_{i+1}=1+y_{i}$.

(3.) The standard model of second-order arithmetic can be characterised up to isomorphism (since every subset of natural numbers is countable, and quasiweak second-order logic has an absolute interpretation for the range of the variables concerning countable relations).

However we can also point out:

Fact. There are models of $\mathbf{Z F C}_{2 Q W}$ in which $\mathbf{C H}$ holds, and models of $\mathbf{Z F C}_{2 Q W}$ in which $\mathrm{CH}$ fails 49

\footnotetext{
${ }^{48} \mathrm{I}$ am grateful to an anonymous reviewer for suggesting the specifics of these examples.

${ }^{49}$ I am grateful to Victoria Gitman for working with me on the following proof:

Proof. Start in a model $\mathfrak{M} \models \mathbf{Z F C}+\neg \mathrm{CH}$ (by preparatory forcing if necessary). Next collapse $\left|\mathcal{P}^{\mathfrak{M}}(\omega)\right|$ to $\omega_{1}$ using the forcing poset $\operatorname{Col}\left(\omega_{1}, \mathcal{P}(\omega)\right)$ in $\mathfrak{M}$. By design, $\mathfrak{M}[G] \models \mathrm{CH}$.
} 
Thus, given the MDA and the Manifestation Thesis, we might think that our current level of informal rigour is manifested by $\mathbf{Z F C}_{2 Q W}$; a logic intermediate between first- and second-order. In this theory, when we can construct an argument for indeterminacy from the MDA we do not have the ability to provide a categorical characterisation, but if no such MDA-style argument is forthcoming (as is the case for the reals under Projective Determinacy) we can characterise many of the relevant structures up to isomorphism.

\section{Objections and replies}

In this section I'll consider some objections and replies. These will not only help to shore up my position, but also will help to see some features of the account.

Objection. What about the Zermelo Categoricity Theorem? One question for the arguments I have posed is immediate: What becomes of the the Zermelo QuasiCategoricity Theorem? One might think that the theorem shows that our thought about the sets is informally rigorous and determines some particular structures (for example those with a specific number of inaccessible cardinals). Earlier, I claimed that it is plausible that there are extensions of our current set concept that support Ultimate- $L$ and and others that support forcing axioms (let's take PFA to make things concrete). I then claimed on the basis of the MDA that our settheoretic discourse and concepts were not informally rigorous. But this is not so (so one might counter-argue) whilst both PFA and Ultimate- $L$ are (let's assume) syntactically consistent with $\mathbf{Z F C} \mathbf{C}_{2}$, only one of them can be true under $\mathbf{Z F C _ { 2 }}$ with the full semantics, the other will require a Henkin-style interpretation to make

But $\mathfrak{M}[G]$ also has the same countable relations on members of $\mathfrak{M}$ as $\mathfrak{M}$ itself, since it is a standard fact about $\operatorname{Col}\left(\omega_{1}, \mathcal{P}(\omega)\right)$ that it is countably closed. (If a countable relation $R$ were added, one can look at the countably many conditions $p_{n} \in \operatorname{Col}\left(\omega_{1}, \mathcal{P}(\omega)\right)$ forcing that $\dot{x} \in \dot{R}$, and (by countable closure) infer that $R$ was already in $\mathfrak{M}$.) Thus $\mathfrak{M}$ and $\mathfrak{M}[G]$ :

(i) Have the same countable relations on sets in $\mathfrak{M}$ (for this reason $\mathfrak{M}$ and $\mathfrak{M}[G]$ have the same reals).

(ii) Differ on the truth value of $\mathrm{CH}$.

Hence $\mathfrak{M}[G]$ thinks that both $\mathfrak{M}$ and $\mathfrak{M}[G]$ satisfy $\mathbf{Z F C}_{2 Q W}$ (since, according to $\mathfrak{M}[G]$, $\mathfrak{M}$ has all its countable relations) but differ on $\mathrm{CH}$. Hence $\mathrm{CH}$ is not fixed by $\mathbf{Z F C}_{2 Q W}$. 
both it and $\mathbf{Z F C}_{2}$ true. So it is just not correct to say that both are legitimate; the concept that motivates a theory that is false under the full semantics requires non-standardness of a certain kind (albeit not as serious as the one required for e.g. $\neg \operatorname{Con}\left(\mathbf{Z F C}_{2}\right)$ ).

The issue here is that this objection assumes that we have access to the range of the second-order variables in making the criticism. We already need to be informal rigorous about the range of second-order variables if we are to hold that $\mathbf{Z F C}_{2}$ is a good encoding of our level of informal rigour. Similar points have been repeatedly stressed throughout the literature ${ }^{50}$, but it is particularly relevant to the current context; a categoricity theorem is meant to encode informal rigour that we have about a certain subject matter, not give us informal rigour (unless we have already accepted some resources for a bottom-up characterisation). If we don't have full informal rigour about set theory (which I've argued for on the basis of the Modal Definiteness Assumption) it is not necessary for us to accept that the categoricity theorem yields genuine clarity ${ }^{51}$

It is instructive here to consider our different interpretations of informal rigour. Isaacson's Kreisel should accept (contrary to what Isaacson claims) that there are different legitimate extensions of our concept of set. This is because for Isaacson's Kreisel, informal rigour is dependent upon the degree to which we have understood a mathematical subject matter. If we expand our concept of set $C_{0}$ to one $C_{1}$ producing a consistent axiomatisation (as, let's assume, both Ultimate- $L$ and PFA do) our understanding should be cashed out in terms of this new concept $C_{1}$, and this determines (given that we are employing $C_{1}$ ) a subject matter that supports either PFA or Ultimate- $L$, depending on which route we

${ }^{50}$ See Meadows (2013) for a survey. Hamkins is also explicit about the point when discussing a version of the categoricity argument in Martin (2001):

The multiversist objects to MartinâĂŹs presumption that we are able to compare the two set concepts in a coherent way. Which set concept are we using when undertaking the comparison? (Hamkins (2012), p. 427)

${ }^{51} \mathrm{~A}$ different move here would be to shift to internal categoricity. If one buys the MDA, however, one will be forced to accept some indeterminacy, blocking the argument to determinacy of $\mathrm{CH}$. For example, even given an internal categoricity argument, indeterminacy in either the range of the first-order quantifiers or in the use of classical logic blocks the argument, in the first case because an internal categoricity result only determines $\mathrm{CH}$ within some restricted first-order domain, and in the second case because the proof of categoricity itself uses classical logic. See Scambler (S) for discussion of this issue. 
pick. Given then that for Isaacson's Kreisel the subject matter we talk about is determined by the concepts we employ, he should accept that we are able to go in different possible directions with our concept, and thus that we are currently not informally rigorous; our set-theoretic discourse is ambiguous between several different sharpenings of the notion.

For exactly the same reason, the Weak Kreiselian Platonist should accept that we are not fully informally rigorous about our concept of set. Recall that for her, informal rigour should be understood as coming to employ ever more platonistically existing precise concepts of set. But for this reason, it's entirely possible that we select one concept that supports PFA in the future and also possible that we select one that supports Ultimate- $L$. In this way, our thinking might be currently ambiguous between several different sharpenings of the concept.

The only person who can argue that the quasi-categoricity theorem in fact shows that $\mathbf{Z F C}{ }_{2}$ encodes our level of informal rigour is the Strong Kreiselian Platonist. They hold that there is a unique correct concept that we are tending towards using informal rigour. This concept can then serve to interpret the secondorder variables, given that $\mathbf{Z F C}_{2}$ is already quasi-categorical. Therefore (they claim) the case as I've set things up is not possible; one of PFA and Ultimate- $L$ (or neither) is correct about this concept, and the process of informal rigour will lead us towards it. Therefore, exactly one or neither of PFA and Ultimate- $L$ is legitimate, and it is just not possible to legitimately expand our concept in incompatible ways and at least one of PFA and Ultimate- $L$ demands a non-full Henkin semantics for its interpretation. Hence, even accepting the MDA we can have informal rigour; simply put there are not incompatible legitimate extensions of our concept.

This represents a coherent position, but not one that I find very plausible due a quadrilemma that I'll develop over the next few pages. The Strong Kreiselian Platonist has to accept that we simply could not coherently follow a different intellectual path from the one we have. But this is an enormously strong claim! What about cases where the kinds of modelling requirements we encounter are very different? Suppose, for example, that there are two physically (or even metaphysically) possible worlds $W_{1}$ and $W_{2}$ at which the modelling requirements for foundations are very different, and $W_{1}$ suggests Ultimate- $L$ where $W_{2}$ suggests PFA. Should we insist that the agents at those worlds with different modelling requirements are doing something illegitimate if they select the 'wrong' concept of set? It seems to me that the agents in the two different cases simply employ different concepts, and use them to talk about different subject matters. But the Strong Kreselian Platonist has to either (a) accept that there is a fundamentally 'correct' interpretation for the second-order variables, and the thinking of one of the two communities' thinking is quite simply flawed, or (b) has to deny that such 
a situation is really possible. I do not find (b) especially plausible since possible worlds are pretty easy to come by. ${ }^{52}$

The situation can be made more vivid by a kind of pessimistic probabilistic argument. Assume that we do have a fully determinate interpretation of $\mathbf{Z F C}$. Notice that it might be that in fact both Ultimate- $L$ and PFA are false in their full generality, even if one is correct about the status of $\mathrm{CH}$. In fact, there are myriad different ways we might develop our set-theoretic axiomatisation, so why should we expect the one we pick to be right? Our understanding of the Generalised Continuum Hypothesis tells us that we can consistently have pretty much whatever pattern we like for the cardinal behaviour of infinite powersets (not to mention a whole gamut of other set-theoretic principles). So, if we believe that there really is a fully determinate $\mathbf{Z F C}_{2}$ model below the first inaccessible, it is overwhelmingly unlikely (without further argument) that we pick exactly the right axiomatisation, and it is we who are saying false things, and can only be interpreted as speaking consistently about non-standard Henkin interpretations.

If, given the Strong Kreiselian Platonist's position, we can coherently justify false set-theoretic principles, we obtain the following further counter-intuitive consequence: We can come to be less precise about the structure we talk about by developing our concept of set and accepting new axioms. Presumably, the Strong Kreiselian Platonist will want to assert that, given an agent $A$ that has come to accept some false axiom(s) $\phi_{0}, \ldots, \phi_{n}$ extending $\mathbf{Z F C}$ that can be satisfied in a transitive model, we should (given a principle of charity) interpret $A$ as saying true things about the relevant transitive models in which $\phi_{0}, \ldots, \phi_{n}$ can be realised, with a Henkin semantics for the relevant second-order variables. This is all well and good when there is an obvious unique model that can be identified as the place to interpret what she says. For example, suppose that we are considering the concept cumulative type structure below the first inaccessible, and further that $A$ believes $V=L$, but (as it turns out) there are non-constructible reals below the first inaccessible. Then, letting $\kappa$ be the least inaccessible, $\left(L_{\kappa}, \in, \mathcal{P}^{L}(\kappa)\right)$ is a natural Henkin model in which to interpret $A^{\prime}$ s discourse, and $A$ has not lost

\footnotetext{
${ }^{52}$ For example Ben-David et al. (2019) showed that a certain learnability problem in machine learning is equivalent to $\mathrm{CH}$. Much of the discussion of this problem (e.g. in Taylor (2019) ) consists of whether or not the algorithms in question are 'real-world' implementable. But if we just have to find some possible world or other rather than the actual world, then these worries about implementation are not so concerning. We can then easily cook-up possible worlds (in some loose sense of possibility) such that in one the evidence points to the learnability of the problem and another in which it points in the other direction.
} 
precision in developing their concept of set to one motivating $V=L$. However, if $\phi_{0}, \ldots, \phi_{n}$ imply that there are unboundedly many measurable cardinals (as many of the candidate extensions of $\mathbf{Z F C}_{2}$ do), then we can point to the following:

Fact. If a theory $\mathbf{T}$ (extending $\mathbf{Z F C} \mathbf{C}_{2}$ and mentioning only set-many parameters) is such that $\mathbf{T} \vdash$ "There are unboundedly many measurable cardinals", then there is no least model of $\mathbf{T}$ under inclusion (within any $\left.\left(V_{\alpha}, \in, \mathcal{P}\left(V_{\alpha}\right)\right)=\mathbf{Z F} \mathbf{C}_{2}\right)$ [3

The core philosophical point is the following: Supposing that the advocate of PFA also accepts the existence of unboundedly-many measurable cardinals, if one of Ultimate- $L$ and PFA+ "There are unboundedly many measurable cardinals" is false, then there is no easily identifiable unique model in which the agent accepting the false theory can be interpreted. Thus, by accepting more axioms on the basis of conceptual refinement (and thus, one might think, becoming more precise about their concept of set) they lose precision concerning the structure they talk about, compared to when they do not accept a refined axiomatisation and stick with $\mathbf{Z F C}_{2}$. This, one might think, is undesirable; we should become more precise, not less precise, by refining our concepts (at least insofar as mathematics is concerned).

Geoffrey Hellman vigorously objects to the conclusion that any of these alternatives undermines the Strong Kreiselian Platonist's position. My arguments are not meant to be knock-down, and indeed one can dig in one's heels here. However, if one does so, one will have to take on one of the following horns of a quadrilemma. Either:

(i) We will not, as a matter of fact, go astray in justifying new axioms extending $\mathbf{Z F C}_{2}$.

Challenge: If we take this horn of the quadrilemma, we then have to explain why we will not go astray in justifying new axioms. This looks like a difficult task and has the whiff of mysticism about it.

\footnotetext{
${ }^{53}$ I thank Monroe Eskew for discussion of the following:
}

Proof. Let $\mathfrak{M}$ be a transitive model of $\mathbf{T}$ and let $\alpha \in \mathfrak{M}$ be such that $\alpha>\operatorname{rank}(a)$ for every parameter $a$ mentioned in $\mathbf{T}$. Let $\kappa$ be a measurable above $\alpha$. Then the embedding induced by the measurability of $\kappa$ produces a proper inner model $\mathfrak{N}$ of $\mathbf{T}$ within $\mathfrak{M}$ (after finding a suitable Henkin interpretation for the second-order variables). Repeating the process yields the conclusion that there is no model of $\mathbf{T}$ least under inclusion contained in $\mathfrak{M}$. If $\mathbf{T}$ does not contain parameters, then one measurable cardinal suffices. 
(ii) We accept $\mathbf{Z F C}_{2}$, but also hold that we cannot justify axioms extending it (except perhaps large cardinals). $\mathbf{Z F C}_{2}$ (possibly with large cardinals added) is the limit of our possible justifications.

Challenge: This option essentially gives up on trying to resolve any sentences that are not consequences of large cardinals (e.g. $\mathrm{CH}$ ).

(iii) An agent can become less precise by refining their set concept (if they pick an axiom with some false consequences).

Challenge: This response seems counter-intuitive; conceptual refinements should result in more rather than less precision.

(iv) One rejects the principle of charity, and accepts that in coming to justify new axioms, we might just say false things about the structure of sets, rather than true things about a range of structures.

Challenge: This likely ascribes a widespread error theory to many attempts to extend $\mathbf{Z F C} \mathbf{C}_{2}$. In particular (given the pessimistic probabilistic argument) it is likely that we will be in error in futures in which we accept a theory resolving questions independent of $\mathbf{Z F C}_{2}$ (plus large cardinals).

It is not impossible to take on one of the horns of the quadrilemma. However, we should contrast the position offered by the Strong Kreiselian Platonist with the alternative offered by either Isaacson's Kreisel or the Weak Kreiselian Platonist. They can argue that whilst we are not yet informally rigorous about our concept of set, and statements like $\mathrm{CH}$ are indeterminate given the concept we employ, we might be informally rigorous in the future. Just look, for example, at the progress that has been made in the hundred years or so since Mirimanoff was writing; our concept of set now clearly underwrites the claim that all sets are well-founded. Perhaps in the future we will come to a fully informally rigorous conception of set on which MDA-style arguments are not possible. However, even in this case we should acknowledge that it is not the case that things had to be this way. Both the Weak Kreiselian Platonist and Isaacson's Kreisel can avoid each of the problems for the Strong Kreiselian Platonist by accepting that what we talk about is partly determined by the axioms we come to justify, and there is no particular 'absolute' interpretation that we are tending towards (or may miss). This difference, whilst it is unlikely to convince the die-hard Strong Kreiselian Platonist, may be dialectically effective for those of us who remain agnostic on the issue, and also presents a challenge for the Strong Kreiselian to explain how they plan on taking on one of the horns.

We can still accept some implications for the quasi-categoricity theorem even given this picture. For, the quasi-categoricity theorem establishes that given an 
interpretation of the second-order variables, a particular structure is identified by $\mathbf{Z F C}{ }_{2}$ (with some specific bound on the inaccessibles) ${ }^{54}$ We might think that this fact has philosophical import. Meadows (in Meadows (2013)) identifies three roles for a categoricity theorem:

(1.) to demonstrate that there is a unique structure which corresponds to some mathematical intuition or practice;

(2.) to demonstrate that a theory picks out a unique structure; and

(3.) to classify different types of theory. (Meadows (2013), p. 526)

He is sceptical about the possibility of (1.) for similar reasons to those I have presented here: The categoricity theorem presupposes the determinateness of the notions it is trying to characterise. However, this is where informal rigour has a role to play; given that we have convinced ourselves of informal rigour, the categoricity theorem tells us that our axiomatisation of this notion has been successful. (2.) is thus important; once we believe we have informal rigour, we need to provide a categorical characterisation to manifest this informal rigour (and ensure that the clarity is genuine). I have argued that for set theory, we are not quite there. However, (3.) is important whether or not we actually have informal rigour. The quasi-categoricity theorem for $\mathbf{Z F C} \mathbf{C}_{2}$, no matter whether or not we are precise about exactly what structures with boundedly many inaccessibles it concerns, does tell us that set theory is non-algebraic. It tells us that our thought at least aims at specifying a particular structure, and hence is not like concepts and theories of general structure (such as that of group) that explicitly aim at dealing with many different non-isomorphic structures. Inside every model of $\mathbf{Z F C}_{2 Q W}$ (which I've argued is possibly the most natural theory for representing our thought about sets), the Zermelo Categoricity Theorem holds and $\mathbf{Z F C} \mathbf{C}_{2}$ (with a specific bound on the inaccessibles) is a theory for talking about one isomorphic structure. It is just that this structure can vary across different models of

${ }^{54}$ Multiversists are often explicit on this point. For example Hamkins writes:

If we make explicit the role of the background set-theoretic context, then the argument appears to reduce to the claim that within any fixed set-theoretic background concept, any set concept that has all the sets agrees with that background concept; and hence any two of them agree with each other. But such a claim seems far from categoricity, should one entertain the idea that there can be different incompatible set-theoretic backgrounds. (Hamkins (2012), p. 427) 
ZFC $_{2 Q W}$. Whilst we are not informally rigorous about set theory, the categoricity theorem shows that this situation is intolerable, there is pressure to become informally rigorous about set theory, even if we currently lack it. This shows that the Difference Thesis (that the case of PP and $\mathrm{CH}$ are fundamentally different from one another) can be retained, even in the face of less than full informal rigour in our set concept.

This observation shows that the distinction between particular and general structures, whilst not incorrect per se, is rather coarse grained. In particular, the idea of general structure further subdivides. First, there are those general structures whose concept does not produce a theory for which there is a categoricity proof (e.g. group), and thus there is no pressure to hold that informal rigour requires us to determine a particular structure. Call these intentionally general structures. There are other concepts (e.g. set below the first inaccessible) where we do have an axiomatisation with a categoricity proof, even if we don't take ourselves to be informally rigorous yet. We call these unintentionally general structures. For set theory, whilst we should not take ourselves to have determined a particular structure, there are still portions of structures corresponding to this concept that are particular (e.g. the representations of some countable structures within models of theories corresponding to our set concept).

Challenge. How do we know when we reach informal rigour? In responding to the last objection, I suggested that there are certain concepts (and discourse) about which we are not yet informally rigorous, but there is nonetheless pressure to become informally rigorous. This immediately raises the following question: How do we know when we are informally rigorous?

My answer here is a little speculative, but it suggests some interesting directions for future research. We begin with the following idea:

Definition. (Informal and Philosophical) We say that a theory T exhibits a highdegree of theoretical completeness when there are no known sentences other than meta-theoretic sentences (e.g. Gödelian diagonal sentences) independent from $\mathbf{T}$.

I acknowledge that this definition is somewhat imprecise. In particular I have no technical account on offer of what is meant by 'meta-theoretic' statements, and I hope that future philosophical research will clarify this notion further. However, it seems that we have some handle on the notion though, there seems to be a sense in which $\operatorname{Con}(\mathbf{Z F C})$ is a statement of a very different kind from $\mathrm{CH}_{55}^{55}$

${ }^{55}$ Not least because $\operatorname{Con}(\mathbf{Z F C})$ is absolute for well-founded models of $\mathbf{Z F C}$, which I've argued our concept of set is sufficient to determine. 
Given a handle upon the notion, I have the following suggestion; a good indicator ${ }^{56}$ of informal rigour is the existence of a categoricity theorem for the relevant second-order theory and a high-degree of theoretical completeness (i.e. the only known sentences independent from our theory are obviously meta-theoretic in some way). If this is the case, then if we take ourselves to be informally rigorous and in fact all known independent statements are meta-theoretic, then we can't construct the kind of simplistic argument from the MDA that I've considered here; any known candidate independent statement does not correspond to a legitimate extension of the concept by design. ${ }^{57}$

This is precisely our current situation in arithmetic. Moreover, as mentioned earlier, if we accept Projective Determinacy (which is agreed on by both Ultimate$L$ and PFA, since they both imply $\left.\mathrm{AD}^{L(\mathbb{R})}\right)$ then the same situation holds for $H\left(\omega_{1}\right)$; the only sentences about $H\left(\omega_{1}\right)$ that are known to be independent from ZFC+PD are meta-theoretic in some way ${ }^{58}$ As we've seen, our current set-theoretic concept lacks this feature for questions at the level of third-order arithmetic and above

\footnotetext{
${ }^{56}$ I stop short of claiming full sufficiency, simply because I'm not clear that these requirements are sufficient and I don't want to overstate my case. The conjecture that replaces "good indicator" with "sufficient" is still worthy of study.

${ }^{57}$ Walter Dean suggests that this part of my view can be seen as a kind of transcendental refutation of the existence of Orey sentences for a given concept. This seems to be precisely what informal rigour should be aiming at; removing the Orey-phenomenon wherever possible by determining a particular structure.

${ }^{58}$ See here Woodin (2001) and Welch (2014), for the point about PD implying a high degree of theoretical completeness for $H\left(\omega_{1}\right)$. See Woodin (2017) and Steel (2005) respectively for the point that Ultimate- $L$ and PFA imply PD. Acceptance of PD is somewhat controversial, and not universally agreed upon. Some (e.g. Barton and Friedman (2017), Barton (2019), Antos et al. (F)) consider versions of the Inner Model Hypothesis (IMH), an axiom candidate relying on extensions of the universe that implies that PD is false. An interesting fact, though one that represents a slight digression (and so I don't include it in the main body of the text) is that (i) variants of this axiom can be coded in strong impredicative class theories (see here Antos et al. (F)) without referring to extensions (other than through coding), and (ii) some of these variants imply that there are no inaccessible cardinals in $V$. A sufficiently strong version of $\mathbf{Z F C}_{2}$ with one of these axioms added would thus be fully (rather than quasi) categorical axiomatisation. The IMH unfortunately does not touch $\mathrm{CH}$ (and so we could still construct the same MDA-style argument), however there are variations of the IMH (e.g. the Strong Inner Model Hypothesis SIMH) that imply that $\mathrm{CH}$ fails badly. Despite these complications, there is a large community of set
} 
(e.g. $\mathrm{CH}$ ). It is this that will enable us to avoid examples of the kind given earlier where we consider two different legitimate concept extensions, since our informally rigorous concept should immediately tell us that one or the other extension is illegitimate. Thus, if my conjecture that a high-degree of theoretical completeness in combination with a categoricity proof is a good-indication of informal rigour, and if we accept $\mathrm{PD}$, and if we accept that we do not have a high-degree of theoretical completeness with respect to set theory, then this supports the idea that $\mathbf{Z F C}_{2 Q W}$ is a good axiomatisation of our current level of informal rigour, since those concepts for which we have a high-degree of theoretical completeness can be determined up to isomorphism, and those which do not cannot.

Of course, given the claim that theoretical completeness in combination with categoricity likely yields informal rigour, our belief in informal rigour is defeasible. It could be, for example, that we discover techniques that allow us to find nonmeta-theoretic sentences independent from our current theories of arithmetic and analysis. Hamkins entertains this suggestion:

My long-term expectation is that technical developments will eventually arise that provide a forcing analogue for arithmetic, allowing us to modify diverse models of arithmetic in a fundamental and flexible way, just as we now modify models of set theory by forcing, and this development will challenge our confidence in the uniqueness of the natural number structure, just as set-theoretic forcing has challenged our confidence in a unique absolute set-theoretic universe. (Hamkins (2012), p. 428)

Perhaps then one thinks that my account goes too far: Surely we should not allow arithmetic to fail to be informally rigorous in such a situation? And what of the situation of the Predicative Iterabilist? Doesn't the possibility of their situation show that in fact our discourse involving the reals is not determinate?

I am quite happy to bite this bullet. If a technique along Hamkins' lines were to be found, I would accept that, after all, our thought concerning arithmetic is not determinate on the basis of the MDA. Given my current evidence however, I find this overwhelmingly unlikely; all such evidence (categoricity, theoretical completeness) seems to indicate that we are informally rigorous, and thus I find it likely that no such technique will be forthcoming.

Even if no such technique concerning the natural numbers is forthcoming, one might object to the idea that our talk concerning the reals is determinate. Recall the case of the Predicative Iterabilist, there we noted that there were different

theorists that do regard PD as well-justified (see Koellner (2014) for a summary) and so I set this point aside for the purposes of this paper. 
legitimate expansions of arithmetic to form the classical and intuitionistic continuum. Doesn't this (on the basis of the MDA) undermine my claim that our concept of real number is indeterminate (and hence $\mathbf{Z F C}_{2 Q W}$ is undermined) $[\sqrt[39]{59}$

I do not find this objection convincing (assuming that we accept Projective Determinacy). Given an utterance of some sentence of the form "the continuum is such that $\phi^{\prime \prime}$ we might:

(a) Implicitly have either the classical or intuitionistic (or maybe even infinitesimal) continuum in mind.

(b) Be using "the continuum" as an algebraic concept to refer to different nonisomorphic continua.

(c) Have a non-informally rigorous concept of continuum which admits of multiple different inconsistent sharpenings.

If we are using "the continuum" in sense (b), then the objection fails to gain traction, since we are only concerned here with informal rigour as it applies to determination of particular structures using non-algebraic theories. The fact that there are algebraic uses of the term "continuum" does not affect the fact that I can be precise when talking about a specific continuum I have in mind in other contexts when employing a different concept (e.g. the classical continuum).

Further, if we are in case (a) the objection also has no force. If, on a given occasion of utterance, I am clear which specific continuum-concept I am employing (say the classical continuum), then the fact that I can use the word "continuum" to apply to other kinds of continua on different occasions is no more problematic than the fact that I can use the word "pingüino" to refer to a delicious chocolate/cream-based snack as well as a kind of flightless bird.

For the objection to have any force, it must be that we are in case (c). Certainly it is plausible that some agents might find themselves in this position, such as the early analysts or even the average student in beginning a first course in analysis. However as far as contemporary research-level mathematics goes, I think there are some reasons to think that we are not in this position concerning the classical continuum. This is because (as mentioned) earlier, if we accept PD (which is agreed on by both proponents of Ultimate- $L$ and forcing axioms) then are no known sentences for which a MDA-style argument could work. Of course, if our confidence in PD were to be challenged (for example by the emergence of a foundational programme rejecting if ${ }^{60}$ then I would be happy to retreat and accept

\footnotetext{
${ }^{59}$ I thank Geoffrey Hellman for pressing this objection.

${ }^{60}$ The Hyperuniverse Programme, which motivates the Inner Model Hypothesis, is plausibly one such programme.
} 
that $\mathbf{Z F C}_{2 W}$ should underwrite our axiomatisation of informal rigour (possibly extended to ensure the well-foundedness of the intended structures). In the other direction, it may turn out in fact that there are agents who are already informally rigorous using $\mathbf{Z F C} \mathbf{C}_{2}$. For example, if we suppose that Ultimate- $L$ comes to be accepted in the next 10 years on the basis of the arguments currently advanced for it, we may wish to conclude that those that currently accept the axiom on these grounds already have a theory of sets with a high-degree of theoretical completeness. For now, I remain agnostic regarding current foundational programmes in the philosophy of set theory, and so find MDA-style arguments at least somewhat convincing.

Objection. Mathematics is necessary! It is very natural at this point to make the following objection: I have claimed that our concept of set is currently not informally rigorous and fails to determine a truth-value for $\mathrm{CH}$. However I've also left open the possibility that in the future we might have an informally rigorous concept of set that determines $\mathrm{CH}$. Moreover, I think that the Axiom of Foundation was not determinate for Mirimanoff's discourse about sets, whereas it is true given our concept of set. But don't I think then that mathematical truth can vary? Doesn't this contradict the widely held assumption that mathematical truth is necessary? My answer: Yes and no. We can have similar discourses using terms like "set" that are interpreted in very different ways at different times. However once the underlying concept of a discipline is fixed, the truths about that concept at that time are necessary. The only way that truth involving the discourse can vary is by the underlying concepts changing somehow ${ }^{61}$ So if by "mathematical truth is necessary" we mean "all truths about every mathematical discourse are fixed" then mathematical truth is not necessary, however if we mean "what is true of particular concepts at particular times is fixed" then mathematical truth is necessary.

A comparison case is useful here. Sheldon Smith (in Smith (2015)) argues (convincingly, in my opinion) that Newton's thought involving the concept derivative could have been sharpened into several precise non-extensionallyequivalent concepts. Two such are the contemporary conception of standard derivative, and the symmetric derivative. For the purposes of our discussion it isn't terribly important how these are defined, but they are not extensionally equivalent (for example, if we consider the absolute value function $f(x)=|x|$, the standard derivative is undefined at the origin, whereas it is the constant 0 function (i.e. the $x$-axis) for the symmetric derivative). Let us suppose (as Smith

\footnotetext{
${ }^{61}$ This idea has much in common with the discussion in Ferreiros (2016) of the idea of invention cum discovery.
} 
argues) that Newton's concept derivative Newton admitted of sharpenings to our concepts standard derivative and symmetric derivative. Then we should hold that Newton's discourse about the derivative of functions did not determine a truth value for the sentence "The derivative of the absolute value function at the origin is the constant 0 function". However, that sentence from our discourse is naturally interpreted (in most contexts) as false, since the concept to be employed (without further specification) for us is standard derivative, and the absolute value function has no derivative at the origin for the standard derivative 62 But we should not think that such an example seriously threatens the idea that mathematical truth is necessary, since the underlying concepts have changed in some way 63

Objection. First-order schemas and second-order interpretations. A key part of Kreisel's 1967 paper is the idea that our commitment to first-order schemas is dependent upon the relevant second-order formulations (e.g. Replacement):

A momentâĂŹs reflection shows that the evidence of the first order axiom schema 64 derives from the second order schema: the difference is that when one puts down the first order schema one is supposed to have convinced oneself that the specific formulae used (in particular, the logical operations) are well defined in any structure that one considers...(Kreisel (1967), p. 148.)

His idea is that the informal rigour about the second-order concept is precisely what motivates the first-order schema. Since we are precise about the relevant particular structure, we can see that the first-order schema is always true on this structure, and this is what justifies the principle. Given this claim, and the fact that I have advocated an indeterminacy in the second-order quantifiers

${ }^{62}$ Thanks here to Zeynep Soysal for suggesting that the concept of derivative might be a pertinent comparison case. See Smith (2015) for the details. That paper also contains several interesting remarks about how we might think conceptual indeterminacy and optimal theories relate in this context, critically examining Rey (1998)'s suggestion that we can implicitly think with a particular concept in virtue of deference to an optimal theory.

${ }^{63}$ Whether or not they are the same concept is a question we leave open and will mention in the conclusion.

${ }^{64}$ Here, Kreisel is in fact talking about induction schema in PA, but the point transfers to Replacement. 
in certain contexts, does this undercut the motivation for the first-order schema of Replacement in terms of its second-order formulations?

Kreisel's point is controversial, but even if we accept the idea my response is quick: No. This is because the motivation for the first-order schema could be interpreted as follows: Given any particular interpretation of the second-order variables (a notion which here I'm taking to be indeterminate) the first-order schema is true. I do not need to be precise about the interpretation of the second-order variables in order to say that however I interpret them, the instances of the firstorder schema hold (this is itself a schematic claim). Kreisel seems to be assuming here that an acceptance of meaningful impredicative second-order theories entails a commitment to determinacy in how the quantifiers are interpreted, but this is a mistake, one can perfectly well accept impredicative second-order theories whilst denying that they have determinate interpretation 65

Objection. You've used notions that are dependent upon a definite concept of set in characterising the debate. A further question is the following: Often I have used phrases like "range of the second-order variables" or "isomorphism" that are naturally interpreted as involving essentially higher-order concepts. But, by my own lights, these notions are indeterminate (for example, I can make two unstructured sets $A$ and $B$ such that $|A|<|B|$ isomorphic by collapsing $|B|$ to $|A|)$. How is this legitimate given that I take our talk about sets to be indeterminate?

There are a two points to make here:

First, I do take myself to be informally rigorous about a good deal of mathematics (for the purposes of this paper anyway). I think it is likely that we, as a community, are informally rigorous about the real numbers and natural numbers, and the concept of well-foundedness. Thus, my view does not collapse into an 'anything goes' relativism.

Second, we can think of this paper as a modelling exercise concerning what we might be able to say about our current thought in the future. I might begin by saying "Suppose that we were informally rigorous about our concept set, what should we then say about our current thought?" I then take myself to have fixed some particular structure $\mathfrak{M}$ about which I am informally rigorous and satisfies $\mathbf{Z F C}_{2}$ (possibly with a Henkin interpretation!) and analyse how the debate might be interpreted relative to $\mathfrak{M}$ (e.g. that from the perspective of this hypothetical fixed universe ${ }^{66}$, our current thought would be best axiomatised by $\mathbf{Z F C}_{2 Q W}$ ).

\footnotetext{
${ }^{65}$ This point has been made increasingly vivid by the recent boom in the study of different class-theoretic systems.

${ }^{66}$ There are options here for how we might interpret this reference. It might be interpreted as picking out a specific such $\mathfrak{M}$ (as outlined in Breckenridge and Magidor (2012))
} 
This will then resemble how our intellectual descendants who are informally rigorous (should there be any) might think of our thought, much as how we now look at Mirimanoff's thought as indeterminate.

\section{Conclusions and open questions}

In this paper, I've argued that there are various foundational programmes and situations we might find ourselves in that support different levels of informal rigour concerning our set-theoretic concepts and thought. In particular, I've suggested that our level of informal rigour in set theory might be insufficient to convince us that our discourse and concepts determine a particular set-theoretic structure. Instead, perhaps we should admit some structural relativity into our characterisations of structures, and a logic weaker than second-order is appropriate for characterising our current thought about sets (in particular $\mathbf{Z F C}_{2 Q W}$ ). I've also argued, however, that there is pressure on us to develop a more informally rigorous concept of set, and thereby answer questions like $\mathrm{CH}$. This identifies a fundamental distinction among the general structures; we have structures that are unintentionally general (like the structure corresponding to our discourse about sets) and those that are intentionally general (like the group structure). This said, there are lots of questions left open by the paper. I take this opportunity to raise some of the main ones.

Question. What is the status of the Modal Definiteness Assumption?

For most of the paper, I was happy to take the MDA as an assumption. I think that given the kinds of possibilities described in the paper (Mirimanoff's futures, and our own) it's a very plausible assumption. This said, I am pretty convinced that both Kreisel and Isaacson would be unhappy with it (since it obviously implies their position concerning the determinateness of $\mathrm{CH}$ is false), and I haven't subjected it to really intense philosophical scrutiny. This is worth examination.

A second question concerns the kinds of particular structures determined by our set-theoretic discourse and concepts. Assuming that I am right that $\mathbf{Z F C}_{2 Q W}$ is the right axiomatisation of our current discourse concerning set theory, there is the question of what is determined on this basis. By and large this theory has not

or an 'arbitrary' such $\mathfrak{M}$ in the style of Fine and Tennant (1983). See Horsten (2019) for a recent treatment. 
(to my knowledge) been studied in detail $[67$ There are some clear candidates for particular structures that can be given categorical characterisations given an acceptance of $\mathbf{Z F C}_{2 Q W}$, we have already mentioned $H\left(\omega_{1}\right)$ and $(\mathbb{R},+, \times,<)$. However, there are others; the Shepherdson-Cohen minimal model for example can be given a categorical axiomatisation, since we can capture absolutely the notion of well-foundedness. The theory consisting of the following axioms:

\section{(i) ZFC-Foundation}

(ii) The Axiom of Foundation formulated as the sentence (in quasi-weak second-order logic) that there are no infinite descending $\in$-chains.

(iii) $V=L$

(iv) $\neg \exists \mathfrak{M}$ “ $\mathfrak{M}$ is a transitive model of $\mathbf{Z F C "}$

identifies a unique model up to isomorphism, since the Shepherdson-Cohen minimal model is (assuming that there is a transitive model of ZFC) the unique transitive model of ZFC satisfying $V=L$ and containing no transitive models of ZFC. An anonymous reviewer helpfully points out that for other countable structures what can be determined up to isomorphism may depend on ambient facts about independence. For example, if we allow non-recursive axiomatisations, a result of Victor Marek states that if there is a projective well-ordering of the reals (e.g. under $V=L$ ) then every countable structure is categorical in second-order logic, and hence also categorical in $\mathbf{Z F C}_{2 Q W}$ (since on countable structures quasiweak second-order logic coincides with full second-order logic). However, this result is independent of $\mathbf{Z F C}$; it is consistent with $\mathbf{Z F C}$ that there are countable ordinals whose second-order theory is not categorical ${ }^{68}$ We therefore ask:

Question. What other structures (both set-theoretic and non-settheoretic) are particular, given that we accept that our thought is axiomatised by $\mathbf{Z F C}_{2 Q W}$, and how can we provide a bottom-up characterisation for them?

${ }^{67}$ Much of what I've considered here was gleaned from Shapiro (2001) and Shapiro (1991). A recent contribution that briefly considers some other versions of ZFC with different underlying logics is Kennedy et al. (S) (esp. §8: Semantic Extensions of ZFC).

${ }^{68} \mathrm{I}$ am very grateful to an anonymous reviewer for explaining these facts and pointing me to the discussion on Mathoverflow at Sáez (2011) and Schweber (2014), as well as the mentioned result in Marek (1973). 
Closely related is whether or not the only unintentionally general structures we talk about are set-theoretic. For all I've said, it might just be set theory that exhibits this feature. We might then ask:

Question. Are there other interesting unintentionally general structures apart from set-theoretic ones?

Throughout the paper, I talked of concepts changing, for example in the shift from Mirimanoff's concept to our own, from Newton's concept of derivative, and from our own concept of set to that of our intellectual descendants. An interesting philosophical question is then in what sense there is a continuity of conceptual content and thought between one intellectual generation and the next. We therefore ask:

Question. When a concept is made more precise, what remains constant, and how should we understand this continuity? Does the concept change or should we rather understand this as a shift to a different concept? Given this, in what sense do we mean the same/similar thing(s) by what we say with our mathematical utterances? 69

We save the toughest question for last. Throughout, I've talked as though we might one day be informally rigorous about our concept of set. However, this might just not be possible. Perhaps any modification of the concept we suggest will be susceptible to decisive objections. Perhaps the different possibilities for extending our concept of set will all seem equally legitimate, and we simply cannot reasonably pick any one concept, whatever the pressure from the quasicategoricity theorem ${ }^{70}$ We therefore ask:

Question. Is it possible for us to legitimately develop an informally rigorous concept of set (at least for each level of the hierarchy)?

\footnotetext{
${ }^{69}$ I am grateful to Chris Scambler for proposing this question and some interesting discussion here. Some possible directions of research (suggested to me by Fenner Tanswell and Juliette Kennedy) include revisiting Lakatos (1976), and in particular development this idea using Waismann's notion of open texture and resources from conceptual engineering. This has been examined in the case of the Church-Turing thesis by Shapiro (2013), but also in the philosophy of mathematics more broadly in Tanswell (2018) and by Vecht $(\mathrm{F})$, with the former providing an application to the universe/multiverse debate..

${ }^{70}$ Considerations along these lines are considered in Hamkins (2012) and Hamkins (2015).
} 
Perhaps we can answer this question affirmatively, or perhaps we are doomed to spend our days like a mathematical version of Buridan's Ass, trapped between equally (un)attractive options. Time will tell.

\section{References}

Aczel, P. (1988). Non-Well-Founded Sets. CSLI Publications.

Antos, C., N. Barton, and S.-D. Friedman (F). Universism and extensions of $V$. Forthcoming in The Review of Symbolic Logic.

Barton, N. (2019, Nov). Forcing and the universe of sets: Must we lose insight? Journal of Philosophical Logic.

Barton, N. and S.-D. Friedman (2017, March). Maximality and ontology: how axiom content varies across philosophical frameworks. Synthese 197(2), 623649.

Ben-David, S., P. Hrubeš, S. Moran, A. Shpilka, and A. Yehudayoff (2019, January). Learnability can be undecidable. Nature Machine Intelligence 1(1), 44-48.

Boolos, G. (1971, April). The Iterative Conception of Set. The Journal of Philosophy 68(8), 215-231.

Breckenridge, W. and O. Magidor (2012). Arbitrary reference. Philosophical Studies 158(3), 377-400.

Button, T. and S. Walsh (2018). Philosophy and Model Theory. Oxford University Press.

Corry, L. (2004). Modern Algebra and the Rise of Mathematical Structures. Birkhäuser.

Dedekind, R. (1888). Was sind und was sollen die Zahlen? In Ewald (1996a), pp. 787-832. Oxford University Press.

Dummett, M. (1977). Elements of Intuitionism. Oxford University Press.

Ewald, W. B. (Ed.) (1996a). From Kant to Hilbert: A Source Book in the Foundations of Mathematics, Volume II. Oxford University Press.

Ewald, W. B. (Ed.) (1996b). From Kant to Hilbert. A Source Book in the Foundations of Mathematics, Volume I. Oxford University Press. 
Feferman, S. and G. Hellman (1995). Predicative foundations of arithmetic. Journal of Philosophical Logic 24(1), 1-17.

Ferreirós, J. (2016). Mathematical Knowledge and the Interplay of Practices. Princeton University Press.

Fine, K. and N. Tennant (1983). A defence of arbitrary objects. Proceedings of the Aristotelian Society 57, 55-77, 79-89.

Gödel, K. (1940). The Consistency of The Continuum Hypothesis. Princeton University Press.

Hamkins, J. D. (2012). The set-theoretic multiverse. The Review of Symbolic Logic 5(3), 416-449.

Hamkins, J. D. (2015). Is the dream solution of the continuum hypothesis attainable? Notre Dame Journal of Formal Logic 56(1), 135-145.

Hekking, J. (2015). Natural models, second-order logic \& categoricity in set theory. Bachelor's Thesis, Leiden University.

Hellman, G. (1996). Structuralism without structures. Philosophia Mathematica 4(2), 100-123.

Hellman, G. and S. Feferman (2000). Challenges to Predicative Foundations of Arithmetic, pp. 317-338. Cambridge University Press.

Horsten, L. (2019). The Metaphysics and Mathematics of Arbitrary Objects. Cambridge University Press.

Incurvati, L. (2014). The graph conception of set. Journal of Philosophical Logic 43(1), 181-208.

Isaacson, D. (2011). The reality of mathematics and the case of set theory. In Z. Noviak and A. Simonyi (Eds.), Truth, Reference, and Realism, pp. 1-75. Central European University Press.

Kanamori, A. (1996). The mathematical development of set theory from Cantor to Cohen. The Bulletin of Symbolic Logic 2(1), 1-71.

Kanamori, A. (2004). Zermelo and set theory. Bulletin of Symbolic Logic 10(4), $487 a ̂ A ̆ S ̧ 553$. 
Kennedy, J., M. Magidor, and J. Väänänen (S). Inner models from extended logics: Part 1. Manuscript under review.

Koellner, P. (2014). Large cardinals and determinacy. In E. N. Zalta (Ed.), The Stanford Encyclopedia of Philosophy (Spring 2014 ed.). Metaphysics Research Lab, Stanford University.

Kreisel, G. (1967). Informal rigour and completeness proofs. In I. Lakatos (Ed.), Problems in the Philosophy of Mathematics, pp. 138-186.

Lakatos, I. (1976). Proofs and Refutations: The Logic of Mathematical Discovery. Cambridge University Press.

Leitgeb, H. (2020, 01). On non-eliminative structuralism. unlabeled graphs as a case study (Part A). Philosophia Mathematica Forthcoming.

Marek, W. (1973). Consistance d'une hypothèse de fraïssé sur la définissabilité dans un language du second ordre. C. R. Acad. Sci. Paris SÃl'r. A-B A-B276, A1147-A1150.

Martin, D. (2001). Multiple universes of sets and indeterminate truth values. Topoi 20(1), 5-16.

McGee, V. (1997). How we learn mathematical language. The Philosophical Review 106(1), 35-68.

Meadows, T. (2013). What can a categoricity theorem tell us? The Review of Symbolic Logic 6, 524-544.

Mirimanoff, D. (1917). Les antinomies de Russell et de Burali-Forti et le probleme fondamental de la theorie des ensembles. L'Enseignement Mathématique 19, 3752.

Reck, E. and G. Schiemer (2019). Structuralism in the philosophy of mathematics. In E. N. Zalta (Ed.), The Stanford Encyclopedia of Philosophy (Winter 2019 ed.). Metaphysics Research Lab, Stanford University.

Resnik, M. (1997). Mathematics as a Science of Patterns. Oxford University Press.

Rey, G. (1998). What implicit conceptions are unlikely to do. Philosophical Issues 9, 93-104.

Rumfitt, I. (2015). The Boundary Stones of Thought: An Essay in the Philosophy of Logic. Oxford University Press. 
Sáez, C. (2011). Categoricity in second order logic. MathOverflow. URL:https://mathoverflow.net/q/72635 (version: 2011-08-10).

Scambler, C. (S). Categoricity and determinacy. Manuscript under review.

Schweber, N. (2014). The (non-)absoluteness of second-order elementary equivalence. MathOverflow. URL:https://mathoverflow.net/q/161676 (version: 2014-03-28).

Shapiro, S. (1991). Foundations without Foundationalism: A Case for Second-order Logic. Oxford University Press.

Shapiro, S. (1997). Philosophy of Mathematics: Structure and Ontology. Oxford University Press.

Shapiro, S. (2001). Systems between First-Order and Second-Order Logics, pp. 131187. Dordrecht: Springer Netherlands.

Shapiro, S. (2013). The open texture of computability. In B. J. Copeland, C. J. Posy, and O. Shagrir (Eds.), Computability: Turing, GÃüdel, Church, and Beyond, pp. 153-181. Cambridge, MA: MIT Press.

Shepherdson, J. (1953). Inner models for set theory-Part III. The Journal of Symbolic Logic 18(2), 145-167.

Shepherdson, J. C. (1951). Inner models for set theory-Part I. Journal of Symbolic Logic 16(3), 161-190.

Shepherdson, J. C. (1952). Inner models for set theory-Part II. Journal of Symbolic Logic 17(4), 225-237.

Smith, S. R. (2015). Incomplete understanding of concepts: The case of the derivative. Mind 124(496), 1163-1199.

Steel, J. R. (2005). PFA implies $A D^{L(\mathbb{R})}$. The Journal of Symbolic Logic 70(4), 12551296.

Tanswell, F. S. (2018). Conceptual engineering for mathematical concepts. Inquiry 61(8), 881-913.

Taylor, W. (2019). Learnability can be independent of zfc axioms: Explanations and implications. 
Väänänen, J. and T. Wang (2015). Internal categoricity in arithmetic and set theory. Notre Dame J. Formal Logic 56(1), 121-134.

Vecht, J. (F). Open texture clarified. Forthcoming in Inquiry, DOI: 10.1080/0020174X.2020.1787222.

Welch, P. (2014). Global reflection principles. Isaac Newton Institute pre-print series, No. NI12051-SAS.

Woodin, H. (2001). The continuum hypothesis, Part I. Notices of the American Mathematical Society 48(6), 569-576.

Woodin, W. H. (2017). In search of Ultimate- $L$ : The 19th Midrasha Mathematicae lectures. The Bulletin of Symbolic Logic 23(1), 1âĂŞ109.

Zermelo, E. (1930). On boundary numbers and domains of sets. In Ewald (1996b), Volume 2, pp. 1208-1233. Oxford University Press. 